\title{
The curvature of branes, currents and gravity in matrix models
}

\author{
Harold Steinacker \\ Faculty of Physics, University of Vienna \\ Boltzmanngasse 5, A-1090 Vienna, Austria
}

\begin{abstract}
The curvature of brane solutions in Yang-Mills matrix models is expressed in terms of conserved currents associated with global symmetries of the model. This implies a relation between the Ricci tensor and the energy-momentum tensor due to the basic matrix model action, without invoking an Einstein-Hilbert term. The coupling is governed by the extrinsic curvature of the brane embedding, which arises naturally for compactified brane solutions. The effective gravity on the brane is thereby related to the compactification moduli, and protected from quantum corrections due to the relation with global symmetries.
\end{abstract}




\section{Contents}

1 Introduction $\quad 2$

2 Matrix models and their geometry 4

2.1 The IKKT model . . . . . . . . . . . . . . . . . . . . . . 4

2.2 Noncommutative branes and their geometry ............ 5

3 Currents and geometry 6

3.1 Currents and conservation laws . . . . . . . . . . . . . . . 7

3.2 Generalized embedding frame . . . . . . . . . . . . . . . . 7

3.3 Connection and curvature . . . . . . . . . . . . . . . . . . 8

3.4 Effective frame . . . . . . . . . . . . . . . . . . . . . . . . . . . . . . . . . . . .

3.5 Special geometry . . . . . . . . . . . . . . . . . . . . . . . . . . . . . . . . . . . . . . . . .

3.6 Curvature and effective gravity . . . . . . . . . . . . . . . 15

3.7 Energy-momentum conservation . . . . . . . . . . . . . . . . 18

3.8 Radial equation of motion and flux stabilization . . . . . . . . . . . 20

4 Perturbations of the geometry 20

4.1 Current conservation and matter ................... 21

5 Conclusion $\quad 22$

$\begin{array}{ll}\text { Appendix A } & 23\end{array}$

$\begin{array}{ll}\text { Appendix B } & 24\end{array}$

$\begin{array}{ll}\text { Appendix C } & 24\end{array}$

$\begin{array}{ll}\text { Appendix D } & 25\end{array}$

$\begin{array}{ll}\text { Appendix E } & 26\end{array}$

\section{Introduction}

Matrix models provide remarkable candidates for a pre-geometric theory of fundamental interactions including gravity. In particular, the IKKT model [1] was proposed originally as a non-perturbative definition of IIB string theory, and the BFSS model ([2], cf. [3]) as a non-perturbative definition of M-theory. It is well-known that the models admit indeed brane solutions, consistent with IIB supergravity resp. 11-dimensional supergravity. However they clearly go beyond supergravity, and should provide a non-perturbative quantum theory by integrating over the space of matrices. In particular the IKKT model is well suited for numerical simulations, and evidence was reported recently [4] for the emergence of 3+1-dimensional space-time. Therefore a better theoretical understanding of the dynamics of branes and their geometry and gravity is very important.

A systematic study of the effective geometry of brane solutions in the matrix model was undertaken in recent years $[5-8]$. This led to a description of branes as quantized symplectic 
submanifold embedded in $\mathbb{R}^{9,1}$, with effective metric $G^{a b} \sim \theta^{a a^{\prime}} \theta^{b b^{\prime}} g_{a^{\prime} b^{\prime}}$ determined by the Poisson structure and the embedding metric $g$. The dynamical metric $G^{a b}$ governs all matter and fields on the branes, and must therefore be interpreted as gravitational metric. The relation with string theory or supergravity is seen by relating $\theta$ with the $B$ field, $g$ with the closed string (bulk) metric, and $G$ with the open string metric on the brane [10].

Since the basic solutions of the model are branes with a dynamical metric, one is led to a picture of brane-worlds. The mechanism for gravity is however not obvious, and there are several possible scenarios. One possibility is that quantization leads to an induced gravity action, which is however delicate and leads to fine-tuning issues. Another mechanism 11 is holography, and indeed the bulk metric of supergravity seems to arise quantum mechanically from the brane description [1, 11 15]. However, to obtain an acceptable 4-dimensional gravity the 10-dimensional bulk must be compactified in this scenario, leading to a landscape of vacua with its inherent lack of predictivity [16].

On the other hand, these conventional pictures miss the basic fact that the metric is not a fundamental degree of freedom in the matrix model, but a derived quantity. This means that the geometrical equations of motion admit solutions of the brane embedding and its Poisson structure given by harmonic brane embeddings and excitations of $\theta^{a b}$. This suggests a different, "emergent" gravity mechanism, based on the basic matrix model action rather than quantum effects. Indeed excitations of the Poisson structure lead to Ricci-flat perturbations [17] on flat $\mathbb{R}^{4}$ and certain self-dual geometries [18]. However on flat branes, matter does not seem to induce the metric perturbations required for gravity. Remarkably, it does on branes with non-trivial extrinsic curvature as pointed out in [19], and indeed Newtonian gravity arises within harmonic brane excitations. A similar mechanism is realized on compactified brane solutions $\mathcal{M}^{4} \times \mathcal{K} \subset \mathbb{R}^{10}$, where the extrinsic curvature arises from the compactification, whose moduli become 4-dimensional gravitational modes [20]. This mechanism is very interesting, but its analysis was limited to the linearized regime, and obscured by certain "mixing" terms whose significance remained unclear.

In this paper, we establish new techniques and results which allow to efficiently compute the curvature of branes in the IKKT model. This provides significant new insights into the above mechanism for emergent gravity. We first establish a description of the geometry in terms of an over-complete frame, based on the currents associated with the global $S O(D)$ symmetry of the model. The curvature can then be computed using techniques from projective modules. We obtain an explicit and compact expression for a broad class of geometries including generalized almost-Kähler geometry, adapted to the case of Minkowski signature. This class of geometries is argued to be sufficiently general and dynamically preferred by the model. The currents are useful because their conservation law encodes the equations of motion of the brane, and moreover the energy-momentum tensor of matter acts as source for currents. Using the results for the curvature, it follows that the energy-momentum tensor couples indeed to the Ricci tensor, albeit in an indirect way mediated by a tensor $\mathcal{P}$ which also provides an additional vacuum contribution. This coupling $\mathcal{P}$ depends on the extrinsic curvature of the brane as well as the Poisson tensor, hence on the brane compactification. Assuming that $\mathcal{P}$ respects the effective 4-dimensional Lorentz symmetry, the Einstein equations should be recovered, up to vacuum contributions. Although more work is required to clarify $\mathcal{P}$ and

\footnotetext{
${ }^{1} \mathrm{~A}$ rather different gravitational interpretation of the IKKT equations of motion was proposed in [21], whose significance for the brane solutions is not clear.
} 
its dynamics, this provides strong support for the emergent gravity scenario on compactified branes $\mathcal{M}=\mathcal{M}^{4} \times \mathcal{K} \subset \mathbb{R}^{D}$ in matrix models.

The relation with global symmetries and with non-commutative gauge theory make this mechanism for gravity very attractive for quantization, for the maximally supersymmetric IKKT model. Since a compactification on fuzzy extra dimensions $\mathcal{K}_{N}$ can be viewed as nontrivial vacuum in a $U(N)$ noncommutative $\mathcal{N}=4 \mathrm{SYM}$ theory, the model is expected to be UV finite on such backgrounds. This is no longer the case for more than 4 noncompact dimensions, which may explain the emergence of $3+1$ non-compact dimensions as observed numerically in [4]. Furthermore, since the currents are associated with global symmetries of the model, the gravitational degrees of freedom can be expected to remain massless at the quantum level, and the mechanism should be protected from fine-tuning problems.

This paper is organized as follows. After reviewing the description of noncommutative branes in matrix models, we introduce the generalized frame formalism for the embedding geometry in section 3.2 based on the currents. This is extended to the effective geometry in section 3.4, where a special class of geometries is introduced. The Ricci tensor for the effective geometry is then computed in section 3.6 . We also discuss the equation of motion for the Poisson structure via energy-momentum conservation in section 3.7, and include a section on flux stabilization. The inhomogeneous current conservation law is derived in section 4, and some technical details are elaborated in the appendices.

\section{Matrix models and their geometry}

We briefly collect the essential ingredients of the matrix model framework and its effective geometry, referring to the review [8] for more details.

\subsection{The IKKT model}

The starting point is given by a matrix model of Yang-Mills type,

$$
S=-\frac{\Lambda_{0}^{4}}{4} \operatorname{Tr}\left(\left[X^{A}, X^{B}\right]\left[X^{C}, X^{D}\right] \eta_{A C} \eta_{B D}+2 \bar{\Psi} \gamma_{A}\left[X^{A}, \Psi\right]\right)=S_{\mathrm{YM}}+S_{\Psi}
$$

where the $X^{A}$ are Hermitian matrices, i.e. operators acting on a separable Hilbert space $\mathcal{H}$. The indices of the matrices run from 0 to $D-1$, and will be raised or lowered with the invariant tensor $\eta_{A B}$ of $S O(D-1,1)$. We also introduce a parameter $\Lambda_{0}$ of dimension $[L]^{-1}$, so that the $X^{A}$ have dimension length. We focus on the maximally supersymmetric IKKT or IIB model [1] with $D=10$, which is best suited for quantization. It is obtained from the $\mathcal{N}=1 U(N)$ SYM on $\mathbb{R}^{10}$ dimensionally reduced to a point, and taking $N \rightarrow \infty$. Then $\Psi$ is a matrix-valued Majorana Weyl spinor of $S O(9,1)$. The model enjoys the fundamental gauge symmetry

$$
X^{A} \rightarrow U^{-1} X^{A} U, \quad \Psi \rightarrow U^{-1} \Psi U, \quad U \in U(\mathcal{H})
$$

as well as the 10-dimensional Poincaré symmetry

$$
\begin{aligned}
& X^{A} \rightarrow \Lambda(g)_{B}^{A} X^{b}, \quad \Psi_{\alpha} \rightarrow \tilde{\pi}(g)_{\alpha}^{\beta} \Psi_{\beta}, \quad g \in \widetilde{S O}(9,1), \\
& X^{A} \rightarrow X^{A}+c^{A} \mathbb{1}, \quad \quad c^{A} \in \mathbb{R}^{10}
\end{aligned}
$$


and a $\mathcal{N}=2$ matrix supersymmetry [1]. The tilde indicates the corresponding spin group. We define the matrix Laplacian as

$$
\square \Phi:=\left[X_{B},\left[X^{B}, \Phi\right]\right]
$$

for any matrix $\Phi \in \mathcal{L}(\mathcal{H})$. Then the equations of motion of the model take the following form

$$
\square X^{A}=\left[X_{B},\left[X^{B}, X^{A}\right]\right]=0,
$$

assuming $\Psi=0$. Analogous statements hold more generally to matrix models of Yang-Mills type, with Euclidean or Minkowski signature.

\subsection{Noncommutative branes and their geometry}

Now we focus on matrix configurations which describe embedded noncommutative (NC) branes. This means that the $X^{A}$ can be interpreted as quantized embedding functions [8]

$$
X^{A} \sim x^{A}: \quad \mathcal{M}^{2 n} \hookrightarrow \mathbb{R}^{10}
$$

of a $2 n$ - dimensional submanifold of $\mathbb{R}^{10}$. More precisely, there should be some quantization map $\mathcal{I}: \mathcal{C}(\mathcal{M}) \rightarrow \mathcal{A} \subset L(\mathcal{H})$ which maps classical functions on $\mathcal{M}$ to a noncommutative (matrix) algebra of functions, such that commutators can be interpreted as quantized Poisson brackets. In the semi-classical limit indicated by $\sim$, matrices are identified with functions via $\mathcal{I}$, and commutators are replaced by Poisson brackets; for a more extensive introduction see e.g. [8, 9]. One can then locally choose $2 n$ independent coordinate functions $x^{a}, a=1, \ldots, 2 n$ among the $x^{A}$, and their commutators

$$
\left[X^{a}, X^{b}\right] \sim i\left\{x^{a}, x^{b}\right\}=i \theta^{a b}(x)
$$

encode a quantized Poisson structure on $\left(\mathcal{M}^{2 n}, \theta^{a b}\right)$. These $\theta^{a b}$ have dimension $\left[L^{2}\right]$ and set a typical scale of noncommutativity $\Lambda_{\mathrm{NC}}^{-2}$. We will assume that $\theta^{a b}$ is non-degenerate 2 , so that the inverse matrix $\theta_{a b}^{-1}$ defines a symplectic form on $\mathcal{M}^{2 n} \subset \mathbb{R}^{10}$. This submanifold is equipped with the induced metric

$$
g_{a b}(x)=\partial_{a} x^{A} \partial_{b} x_{A}
$$

which is the pull-back of $\eta_{A B}$. However, this is not the effective metric on $\mathcal{M}$. To understand the effective metric and gravity, we need to consider matter on the brane $\mathcal{M}$. Bosonic matter or fields arise from nonabelian fluctuations of the matrices around a stack $X^{A} \otimes \mathbb{1}_{n}$ of coinciding branes, while fermionic matter arises from $\Psi$ in (2.1). It turns out that in the semi-classical limit, the effective action for such fields is governed by a universal effective metric $G^{a b}$. It can be obtained most easily by considering the action of an additional scalar field $\phi$ coupled to the matrix model in a gauge-invariant way, with action

$$
\begin{aligned}
S[\phi] & =-\frac{\Lambda_{0}^{4}}{2} \operatorname{Tr}\left[X_{A}, \phi\right]\left[X^{A}, \phi\right] \sim \frac{\Lambda_{0}^{4}}{2(2 \pi)^{n}} \int d^{2 n} x \sqrt{\left|\theta^{-1}\right|} \theta^{a a^{\prime}} \theta^{b b^{\prime}} g_{a^{\prime} b^{\prime}} \partial_{a} \phi \partial_{b} \phi \\
& =\frac{\Lambda_{0}^{4}}{2(2 \pi)^{n}} \int d^{2 n} x \sqrt{|G|} G^{a b} \partial_{a} \phi \partial_{b} \phi .
\end{aligned}
$$

\footnotetext{
${ }^{2}$ If the Poisson structure is degenerate, then the fluctuations propagate only along the symplectic leaves.
} 
Therefore the effective metric is given by [6]

$$
\begin{aligned}
& G^{a b}=e^{-\sigma} \gamma^{a b}, \quad \gamma^{a b}=\theta^{a a^{\prime}} \theta^{b b^{\prime}} g_{a^{\prime} b^{\prime}} \\
& e^{-\sigma}=\left(\frac{\operatorname{det} \theta_{a b}^{-1}}{\operatorname{det} G_{a b}}\right)^{\frac{1}{2}}=\left(\frac{\operatorname{det} \theta_{a b}^{-1}}{\operatorname{det} g_{a b}}\right)^{\frac{1}{2(n-1)}} .
\end{aligned}
$$

It is useful to consider the conformally equivalent metri $33 \gamma^{a b}$ which satisfies

$$
\sqrt{\left|\theta^{-1}\right|} \gamma^{a b}=\sqrt{|G|} G^{a b}
$$

The effective metric $G^{a b}$ is encoded in the matrix Laplace operator, which can be seen from the following result [8] for the semi-classical limit

$$
\square \Phi=\left[X_{A},\left[X^{A}, \Phi\right]\right] \sim-e^{\sigma} \square_{G} \phi
$$

acting on scalar fields $\Phi \sim \phi$. In particular, the matrix equations of motion (2.5) take the simple form $\square X^{A} \sim-e^{\sigma} \square_{G} x^{A}=0$. This means that the embedding functions $x^{A} \sim X^{A}$ are harmonic functions with respect to $G$. Furthermore, the bosonic part of the matrix model action (2.1) can be written in the semi-classical limit as follows

$$
S_{\mathrm{YM}} \sim \frac{\Lambda_{0}^{4}}{4(2 \pi)^{2 n}} \int d^{2 n} x \sqrt{\left|\theta^{-1}\right|} \gamma^{a b} g_{a b}
$$

Compactified brane solutions. Of particular interest here are branes with compactified extra dimensions

$$
\mathcal{M}^{2 n}=\mathcal{M}^{4} \times \mathcal{K} \quad \subset \mathbb{R}^{D}
$$

where the extrinsic curvature is predominantly due to $\mathcal{K} \subset \mathbb{R}^{D}$, while the embedding of $\mathcal{M}^{4}$ is approximately flat. Such solutions including $\mathcal{K}=T^{2}$ and $\mathcal{K}=S^{3} \times S^{1}$ have been given recently [22], where $\mathcal{K}$ is rotating along $\mathcal{M}^{4}$ and stabilized by angular momentum. This is possible because of "split noncommutativity", where the Poisson structure relates the compact space $\mathcal{M}^{4}$ with the non-compact space $\mathcal{K}$,

$$
\theta^{\mu i}=\left\{x^{\mu}, y^{i}\right\} \neq 0 .
$$

Here $x^{\mu}$ are coordinates on $\mathcal{M}^{4}$ and $y^{i}$ are coordinates on $\mathcal{K}$. As pointed out in [20], such a structure relates the perturbations of $\mathcal{K}$ to perturbations of the effective metric on $\mathcal{M}^{4}$, and thereby links the Ricci tensor to the energy-momentum tensor. This leads to a novel mechanism for 4-dimensional gravity. The aim of this paper is to understand better this mechanism, by computing the intrinsic curvature on the brane in the presence of matter.

\section{Currents and geometry}

To compute the curvature of $\mathcal{M}$ directly from the metric $\gamma_{a b}$ is complicated and not illuminating. We will develop a suitable generalized frame formalism, which allows to express the curvature efficiently in terms of the $S O(D)$ conserved currents. This will be the key to a better understanding of the effective gravity on the branes.

\footnotetext{
${ }^{3}$ More abstractly, this can be stated as $(\alpha, \beta)_{\gamma}=\left(i_{\alpha} \theta, i_{\beta} \theta\right)_{g}$ where $\theta=\frac{1}{2} \theta^{a b} \partial_{a} \wedge \partial_{b}$.
} 


\subsection{Currents and conservation laws}

The matrix model (2.1) is invariant under the $S O(D)$ resp. $S O(1, D-1)$ symmetries 4

$$
\delta X^{A}=\left(\lambda^{\alpha}\right)_{B}^{A} X^{B}
$$

for $\lambda^{\alpha} \in \mathfrak{s o}(D)$. Setting $\Psi=0$ for now, they lead to conserved currents in complete analogy to quantum field theory,

$$
\left[X^{A}, \tilde{J}_{A}^{\alpha}\right]=0, \quad \tilde{J}_{A}^{\alpha}=\frac{1}{2} \lambda_{C D}^{\alpha}\left\{X^{C},\left[X_{A}, X^{D}\right]\right\}
$$

with anti-symmetric $\lambda_{A B}^{\alpha} \in \mathfrak{s o}(D)$. This can be verified directly using the equation of motion (2.5), or more conceptually via a matrix version of the Noether theorem, as elaborated in appendix A. In the semi-classical limit, this reduces to

$$
\begin{aligned}
\nabla^{a} J_{a}^{\alpha} & =0 \quad J_{a}^{\alpha}=x^{A} \lambda_{A B}^{\alpha} \partial_{a} x^{B} \\
\tilde{J}_{A}^{\alpha} & \sim i \theta^{a b} \partial_{a} x_{A} J_{b}^{\alpha}
\end{aligned}
$$

where $\nabla$ is the Levi-Civita connection corresponding to the effective metric $G$. The conservation law in the presence of matter will be discussed in section 4.1. These conservation laws completely capture the equation of motion for the modes which preserve $S^{D-1} \subset \mathbb{R}^{D}$. Due to their origin from global symmetries, these conservation laws are expected to be protected from quantum corrections, as usual in quantum field theory. This makes them well suited to describe the geometry of the model and its dynamics.

\subsection{Generalized embedding frame}

The above currents are naturally viewed as one-forms in the cotangent bundle $T^{*} \mathcal{M}$,

$$
J^{\alpha}=x^{A} \lambda_{A B}^{\alpha} d x^{B}=J_{a}^{\alpha} d \xi^{a}, \quad J_{a}^{\alpha}=x \lambda^{\alpha} \partial_{a} x
$$

(dropping the $\mathbb{R}^{D}$ indices) where $\lambda^{\alpha}=\lambda_{A B}^{\alpha} \in \mathfrak{s o}(D)$, and $\xi^{a}$ denotes any local coordinates on $\mathcal{M}$. It is useful to supplement them with the "radial" current corresponding to $\lambda^{0}=\mathbb{1}$,

$$
J_{a}^{0}=x \lambda^{0} \partial_{a} x, \quad J^{0}=x_{A} d x^{A}=J_{a}^{0} d \xi^{a}=r d r
$$

where

$$
r^{2}=x_{A} x^{A}
$$

is the invariant radius on $\mathbb{R}^{D}$. The basic observation underlying this paper is that these currents provide a generalized, over-complete frame for the metric $g$. More precisely, define the one-forms

$$
\theta_{a}^{\alpha}=r^{-1} J_{a}^{\alpha}, \quad \theta^{\alpha}=r^{-1} J^{\alpha} .
$$

\footnotetext{
${ }^{4}$ When we write $S O(D)$ usually $S O(1, D-1)$ will also be understood.
} 
Then the following identity 5 holds

$$
g_{a b}=\kappa_{\alpha \beta} \theta_{a}^{\alpha} \theta_{b}^{\beta}=r^{-2} \kappa_{\alpha \beta} J_{a}^{\alpha} J_{b}^{\beta} .
$$

where

$$
\kappa^{\alpha \beta}=\left(\begin{array}{cc}
1 & 0 \\
0 & -\frac{1}{2} \operatorname{tr} \lambda^{\alpha} \lambda^{\beta}
\end{array}\right)
$$

is the Killing form of $\mathfrak{s o}(D)$ resp. $\mathfrak{s o}(1, D-1)$ supplemented by $\lambda^{0}$. This can be seen using the identity

$$
\kappa_{\alpha \beta} \lambda_{A B}^{\alpha} \lambda_{C D}^{\beta}=\eta_{A C} \eta_{B D}-\eta_{A D} \eta_{B C}+\eta_{A B} \eta_{C D},
$$

which is easy to check for the basis of $\lambda^{\alpha}$ given by

$$
\lambda_{C D}^{(A B)}=\delta_{C}^{A} \delta_{D}^{B}-\delta_{D}^{A} \delta_{C}^{B}, \quad A<B \quad \text { and } \quad \lambda_{C D}^{0}=\eta_{C D}
$$

where $\kappa^{\alpha \beta}=\delta^{\alpha \beta}$ in the Euclidean case. Correspondingly,

$$
P^{\alpha \beta}=\theta_{a}^{\alpha} \theta_{b}^{\beta} g^{a b}, \quad P_{\beta}^{\alpha} \theta^{\beta}=\theta^{\alpha}
$$

is a projector on the cotangent bundle $T^{*} \mathcal{M}$; the frame indices will always be raised and lowered with $\kappa_{\alpha \beta}$, e.g. $P_{\beta}^{\alpha}=P^{\alpha \gamma} \kappa_{\beta \gamma}$. The projector on the normal bundle is then given by

$$
P_{N}^{\beta \gamma}=\kappa^{\beta \gamma}-P^{\beta \gamma}, \quad\left(P_{N}\right)_{\beta}^{\alpha} \theta^{\beta}=0 .
$$

Since the frame $\theta^{\alpha}$ is (over-) complete, any one-form on $\mathcal{M}$ can be written as $v=\sum_{\alpha} \theta^{\alpha} v_{\alpha}=$ $\sum_{\alpha} \theta^{\alpha} P_{\alpha}{ }^{\beta} v_{\beta}$. This expansion is unique if we impose that $P v=v$. In other words, the space of one-forms on $\mathcal{M}$ can be identified with the projective module $6 \Omega^{1}(\mathcal{M}) \cong \mathcal{E}_{g}:=P \mathcal{A}^{N}$, where $\mathcal{A}=\mathcal{C}(\mathcal{M})$. This construction turns out to be very useful to compute the curvature.

Furthermore, the following identity is shown in appendix B;

$$
d \theta^{\beta} P_{\beta}^{\alpha}=-\theta^{\beta} \omega_{\beta}^{\alpha}
$$

where

$$
\omega^{\beta \alpha}=r^{-1}\left(\theta^{\beta} P^{0 \alpha}-P^{0 \beta} \theta^{\alpha}\right)=-\omega^{\alpha \beta} .
$$

which satisfies $P \omega=\omega=\omega P$.

\subsection{Connection and curvature}

The above realization of the cotangent bundle as as projective module $T^{*} \mathcal{M} \cong \mathcal{E}_{g}$ is useful, because it provides a canonical ("Grassmann") connection

$$
\nabla_{g}=P \circ d: \quad \mathcal{E}_{g} \rightarrow \mathcal{E}_{g} \otimes_{\mathcal{A}} \Omega^{1}(\mathcal{A})
$$

\footnotetext{
${ }^{5}$ No equation of motion or current conservation is needed here.

${ }^{6}$ The label $\mathcal{E}_{g}$ indicates that the frame $\theta^{\alpha}$ encodes the metric $g$, to distinguish it from $\mathcal{E}_{\gamma}$ introduced below. Module means that the elements can be multiplied (from the right, most naturally) with functions $f \in \mathcal{A}$.
} 
The curvature of this connection is defined by

$$
\mathcal{R}[g]=\nabla_{g}^{2}=P d P d P
$$

for an introduction to these concepts see e.g. [23]. The last identity follows using $\theta=P \theta$ from

$$
\nabla_{g}^{2} \psi=P d(P d(P \psi))=(P d P d P) \psi
$$

Under gauge transformations $v \rightarrow \Lambda v \in \mathcal{E}$ which commute with $P$, the connection transforms as $\nabla_{g} \rightarrow \nabla_{g}^{\prime}=\Lambda \nabla_{g} \Lambda^{-1}=\nabla_{g}+P \Lambda^{-1} d \Lambda$, and the curvature $\nabla_{g}^{2}=P d P d P$ transforms in the adjoint. Furthermore, $\nabla_{g}$ is compatible with the inner product on $\mathcal{E}_{g}$ which arises from $\kappa^{\alpha \beta}$ restricted to $\mathcal{E}=P \mathcal{A}^{N}$,

$$
d(v, w)_{g}=\left(\nabla_{g} v, w\right)_{g}+\left(v, \nabla_{g} w\right)_{g}
$$

where

$$
(v, w)_{g}=v w^{\dagger}=v_{\alpha} \kappa^{\alpha \beta} w_{\beta}=v_{\alpha} P^{\alpha \beta} w_{\beta}=v_{a} g^{a b} w_{b}
$$

because $P w=w, P v=v$. This is the usual metric compatibility condition. The gauge transformations are compatible with this inner product if $\Lambda^{\dagger}=\Lambda^{-1}$, where

$$
\left(v^{\dagger}\right)^{\alpha}=\kappa^{\alpha \beta} v_{\beta}, \quad\left(\Lambda^{\dagger}\right)_{\beta}^{\alpha}=\kappa_{\delta \beta} \Lambda_{\gamma}^{\delta} \kappa^{\alpha \gamma}
$$

etc. Finally, the torsion $T: \mathcal{E} \cong \Omega^{1}(\mathcal{A}) \rightarrow \Omega^{2}(\mathcal{A})$ is defined a: ${ }^{7}$

$$
T(v f)=(d+m \circ \nabla)(v f)=T(v) f
$$

where $m(\theta \otimes \alpha)=\theta \wedge \alpha$.

Let us compute the Grassmann connection explicitly. The one-form $\theta^{\beta}$ is represented by

$$
\theta^{\beta}=\theta^{\alpha} P_{\alpha}{ }^{\beta} \cong\left(P_{\alpha}^{\beta}\right) \quad \in \mathcal{E}=P \mathcal{A}^{N}
$$

and its covariant derivative is

$$
\nabla_{g} \theta^{\beta}=\theta^{\alpha} \otimes\left(P_{\alpha}^{\gamma} d P_{\gamma}^{\beta}\right)
$$

Therefore $P d P$ is the connection coefficient. The torsion is given by

$$
\begin{aligned}
T^{\gamma} & =d \theta^{\gamma}+m\left(\nabla_{g} \theta^{\gamma}\right)=d \theta^{\gamma}+\theta^{\alpha} \wedge P_{\alpha}^{\beta} d P_{\beta}^{\gamma} \\
& =d\left(\theta^{\beta} P_{\beta}^{\gamma}\right)+\theta^{\beta} \wedge d P_{\beta}^{\gamma} \\
& =d \theta^{\beta} P_{\beta}^{\gamma}=-\theta^{\beta} \omega_{\beta}^{\gamma}
\end{aligned}
$$

using 8 the result (3.14). More general connections can be defined as $\nabla \rightarrow \nabla+A$ where $A=A^{\alpha}{ }_{\beta} \in \Omega^{1}(\mathcal{A})$ such that $P A=A=A P$, which is metric compatible if $A^{\dagger}=-A$. In particular, the torsion vanishes if we choose $A=\omega$,

$$
0=T^{\alpha}[g]=T^{\alpha}+\theta^{\beta} \wedge \omega_{\beta}^{\alpha}=0 .
$$

\footnotetext{
${ }^{7}$ The present definitions entail $m \circ \nabla(v f)=\nabla(v) f+v \wedge d f$ so that gauge covariance holds.

${ }^{8}$ Note that $\omega$ is not the spin connection, and the curvature is not given by $d \omega+\omega \omega$.
} 
This is compatible with the metric since $\omega^{\dagger}=-\omega$ (3.15), and therefore $\nabla[g]=\nabla_{g}+\omega$ is the Levi-Civita connection on $\mathcal{M}$ for $g$.

The curvature 2 -form $\mathcal{R}_{\beta}^{\alpha}$ is a linear map on $\mathcal{E}_{g} \cong T^{*} \mathcal{M}$, which can be written in standard tensorial form using the frame. The Grassmann curvature can be evaluated easily noting that $\theta P_{N}=0$ along with $d P=-d P_{N}$ and $P^{\dagger}=P$ :

$$
\begin{aligned}
\mathcal{R}[g] & =\nabla[g]^{2}=P d P d P \\
\mathcal{R}_{a c}[g] & =\theta_{a} \mathcal{R} \theta_{c}^{\dagger}=\theta_{a} d P_{N} d P_{N} \theta_{c}^{\dagger}=d \theta_{a} P_{N} d \theta_{c}^{\dagger} \\
& =r^{-2} \partial_{e} J_{a}^{\beta}\left(P_{N}\right)_{\beta \gamma} \partial_{f} J_{c}^{\gamma} d x^{e} d x^{f}
\end{aligned}
$$

using $d \theta P_{N}=r^{-1} d J P_{N}$ in the last step.

Normal embedding coordinates (NEC). To evaluate this, we first choose suitable coordinates at any given point $p \in \mathcal{M}$ : using the rotation symmetry of the model, we can assume that $T_{p} \mathcal{M} \cong\left(\mathbb{R}^{2 n}, 0, \ldots, 0\right)$. We can then choose the first $2 n$ matrix components $x^{a}$ as local coordinates, denoted as "normal embedding coordinates" NEC. It follows that $\partial x_{A} \partial \partial x^{A}=0$ at $p \in \mathcal{M}$, which implies $\left.\partial\right|_{p} g_{a b}=0$. Therefore these are indeed normal coordinates for $g$ in the Riemannian sense, so that we are essentially using $\nabla[g]$. We can furthermore assume $p=\left(0, \ldots, 0, r_{0}\right)$ after a suitable translation, so that

$$
\left.x_{A} \partial x^{A}\right|_{p}=\left.r \partial r\right|_{p}=\left.J^{0}\right|_{p}=0 .
$$

Now consider the following tensors

$$
\begin{aligned}
T_{a b}^{\alpha} & =\partial_{a} x \lambda^{\alpha} \partial_{b} x \\
K_{a b}^{\alpha} & =x \lambda^{\alpha} \nabla_{a}[g] \partial_{b} x=\left.x \lambda^{\alpha} \partial_{a} \partial_{b} x\right|_{p}=K_{b a}^{\alpha},
\end{aligned}
$$

in particular $T_{a b}^{0}=g_{a b}$. Clearly $K_{a b}^{\alpha}$ characterizes the exterior curvature of $\mathcal{M} \subset \mathbb{R}^{D}$. Then

$$
\nabla_{a}[g] J_{b}^{\alpha}=T_{a b}^{\alpha}+K_{a b}^{\alpha}=\left.\partial_{a} J_{b}^{\alpha}\right|_{p},
$$

We note that $T_{a b}^{\alpha}=-T_{b a}^{\alpha}$ for $\alpha \neq 0$. It follows using (3.10) that

$$
\begin{aligned}
K_{e a}^{\alpha} \kappa_{\alpha \beta} J_{d}^{\beta} & =0=K_{e a}^{\alpha} P_{\alpha}^{\beta} \\
T_{e a}^{\alpha} \kappa_{\alpha \beta} J_{d}^{\beta} & =J_{e}^{0} g_{a d}-J_{a}^{0} g_{e d}+J_{d}^{0} g_{e a} \\
\left.T_{e a}^{\alpha} P_{\alpha}^{\beta}\right|_{p} & =0=\left.T_{e a}^{\alpha} P_{\alpha \beta} T_{f c}^{\beta}\right|_{p} .
\end{aligned}
$$

Therefor $9 K^{\alpha}$ and $T^{\alpha}$ live in the normal bundle at $p$, and dropping the contributions of $J^{0}$ at $p$ the Grassmann curvature 2-form for the metric $g$ is

$$
\mathcal{R}[g]_{a c}=r^{-2} \nabla_{e} J_{a} \nabla_{f} J_{c}^{\dagger} d x^{e} d x^{f}
$$

where $\nabla=\nabla[g]$. The point is that the Grassmann curvature tensor can be expressed in terms of the $\mathfrak{s o}(D)$ currents of the matrix model. We now obtain the Riemann tensor for $g$ via

$$
\begin{aligned}
R[g] & =\nabla[g]^{2}=P d P d P+P(d \omega+\omega \omega) P \\
R_{a c}[g] & =\mathcal{R}_{a c}[g]+\theta_{a}(d \omega+\omega \omega) \theta_{c}^{\dagger} \equiv \mathcal{R}_{a c}[g]+\mathcal{R}_{a c}[\omega] .
\end{aligned}
$$

\footnotetext{
${ }^{9}$ Note that $\left(\nabla_{a} J_{b}\right) J_{c}^{\dagger}$ does notvanish identically. This is the reason why the final result (3.34) contains additional radial contributions $\nabla J^{0}$, and is not obtained trivially by re-shuffling $\nabla J \nabla J$.
} 
Here $\omega$ takes care of the radial contributions which are not captured by the $\mathfrak{s o}(D)$ currents, as computed in appendix $\mathbb{C}$. Together with the above we obtain

$$
R[g]_{a c}=r^{-2}\left(\nabla_{e} J_{a} \nabla_{f} J_{c}^{\dagger}-g_{a e} \nabla_{f} J_{c}^{0}-g_{c f} \nabla_{e} J_{a}^{0}\right) d x^{e} d x^{f}
$$

where $\nabla=\nabla[g]$, dropping again contributions of $\left.J^{0}\right|_{p}=0$ and recalling $\nabla_{a} J_{b}^{0}=g_{a b}+K_{a b}^{0}=$ $\frac{1}{2} \nabla_{a} \partial_{b} r^{2}$. This is the key result, which will be extended to the effective metric $\gamma$ in the next section. As a check, we proceed by decomposing $\nabla_{a} J_{b}^{\alpha}=T_{a b}^{\alpha}+K_{a b}^{\alpha}$ and using

$$
r \nabla_{a} \partial_{b} r=\frac{1}{2} \nabla_{a} \partial_{b} r^{2}=\partial_{a} x_{A} \partial_{b} x^{A}+x_{A} \nabla_{a} \partial_{b} x^{A}=g_{a b}+x_{A} \nabla_{a} \partial_{b} x^{A}
$$

along with the identity (3.10) to obtain

$$
\begin{aligned}
T_{e a}^{\alpha} \kappa_{\alpha \beta} T_{f c}^{\beta} & =\left(\partial_{e} x \lambda^{\alpha} \partial_{a} x\right)\left(\partial_{f} x \lambda_{\alpha} \partial_{c} x\right)=g_{e f} g_{a c}-g_{e c} g_{a f}+g_{a e} g_{c f} \\
K_{e a}^{\alpha} \kappa_{\alpha \beta} T_{f c}^{\beta} & =\left(x \lambda^{\alpha} \nabla_{e} \partial_{a} x\right)\left(\partial_{f} x \lambda_{\alpha} \partial_{c} x\right)=K_{e a}^{0} g_{f c} \\
T_{e a}^{\alpha} \kappa_{\alpha \beta} K_{f c}^{\beta} & =\left(\partial_{e} x \lambda_{\alpha} \partial_{a} x\right)\left(x \lambda^{\alpha} \nabla_{f} \partial_{c} x\right)=g_{e a} K_{f c}^{0} \\
K_{e a} P_{N} K_{f c} & =K_{e a}^{\alpha} \kappa_{\alpha \beta} K_{f c}^{\beta}=r^{2} \nabla_{e} \partial_{a} x_{A} \nabla_{f} \partial_{c} x^{A} .
\end{aligned}
$$

Since we assumed NEC, the coordinate-invariant form is obtained by replacing $\partial_{a} \rightarrow \nabla_{a}[g]$. We thus recover the usual Gauss-Codazzi theorem 10 for the Riemann curvature tensor on $\mathcal{M} \subset \mathbb{R}^{D}$

$$
R[g]_{a c}=d \partial_{a} x_{A} d \partial_{c} x^{A}=\frac{1}{2}\left(\nabla_{e} \partial_{a} x_{A} \nabla_{f} \partial_{c} x^{A}-\nabla_{f} \partial_{a} x_{A} \nabla_{e} \partial_{c} x^{A}\right) d x^{e} d x^{f}
$$

\subsection{Effective frame}

We now want to develop a similar machinery for the effective metric $\gamma_{a b}$ on $\mathcal{M}$. This metric is encoded in the following (over-complete) tangent frame associated to the currents,

$$
\begin{aligned}
V^{\alpha} & =x \lambda^{\alpha}\{x, .\}=J_{a}^{\alpha} \theta^{a b} \partial_{b} \quad \in T \mathcal{M} \\
V^{\alpha} V^{\beta} \kappa_{\alpha \beta} & =r^{2} \gamma^{a b} \partial_{a} \otimes \partial_{b}
\end{aligned}
$$

including $\alpha=0$ as before. Here $\{.,$.$\} is the Poisson bracket on \mathcal{M}$, which arises from the non-commutative nature of the brane. However to compute the curvature, it is more natural to use the corresponding frame of one-forms, defined as usual by lowering the index with the effective metric $\gamma$. Thus

$$
\Theta^{\alpha}=\Theta_{a}^{\alpha} d \xi^{a}, \quad \Theta_{a}^{\alpha}=r^{-1} V^{\alpha, b} \gamma_{b a}=\theta_{b}^{\alpha} \mathcal{J}_{a}^{b}
$$

where

$$
\mathcal{J}_{a}^{c}=\theta^{c b} \gamma_{b a}=\theta_{a b}^{-1} g^{b c}
$$

Then the effective metric can be written as

$$
\gamma_{a b}=\kappa_{\alpha \beta} \Theta_{a}^{\alpha} \Theta_{b}^{\beta}=g_{a^{\prime} b^{\prime}} \mathcal{J}_{a}^{a^{\prime}} \mathcal{J}_{b}^{b^{\prime}}=-g_{a c}\left(\mathcal{J}^{2}\right)^{c}{ }_{b}
$$

\footnotetext{
${ }^{10}$ This can be obtained quickly using the projective module defined by the over-complete frame $\theta^{A}=d x^{A}$.
} 
and the tangential projector can be expressed in various ways

$$
\begin{aligned}
P^{\alpha \beta} & =\Theta_{a}^{\alpha} \Theta_{b}^{\beta} \gamma^{a b}=\theta_{c}^{\alpha} \theta_{d}^{\beta} \mathcal{J}_{a}^{c} \mathcal{J}_{b}^{d} \gamma^{a b}=\theta_{a}^{\alpha} \theta_{b}^{\beta} g^{a b}=-\Theta_{a}^{\alpha} \theta^{a e} \theta_{e}^{\beta} \\
P^{\alpha \beta} \Theta^{\beta} & =\Theta^{\alpha}, \quad P^{\alpha \beta} \theta^{\beta}=\theta^{\alpha} .
\end{aligned}
$$

Note that $P$ coincides with the projector defined in the previous section; this is evident due to the relation (3.39) between the frames. The symplectic form $\Omega$ on $\mathcal{M}$ is then given by

$$
\Theta_{a} \theta_{b}^{\dagger}=\theta_{c} \mathcal{J}_{a}^{c} \theta_{b}^{\dagger}=\theta_{a b}^{-1}, \quad \Theta \theta^{\dagger}=\Omega .
$$

A cotangent vector can now be written in the two bases as $v=\theta^{\alpha} v_{\alpha}=\Theta^{\alpha} v_{\alpha}^{\prime}$ with $P v=$ $v, P v^{\prime}=v^{\prime}$. This gives two different identifications of $T^{*} \mathcal{M}$ with projective modules $\mathcal{E}_{g}$ resp. $\mathcal{E}_{\gamma}$. We can determine the transformation $\Lambda v^{\prime}=v$ between the two frames explicitly, such that

$$
\Theta^{\alpha}=\theta^{\beta} \Lambda_{\beta}^{\alpha}
$$

and therefore

$$
\gamma_{a b}=\Theta_{a} \Theta_{b}^{\dagger}=\theta_{a} \Lambda \Lambda^{\dagger} \theta_{b}^{\dagger} .
$$

This $\Lambda$ is of course not unique. A nice invertible $\Lambda$ which satisfies this requirement is given by

$$
\begin{aligned}
\Lambda^{\alpha \beta} & =P_{N}^{\alpha \beta}+\theta_{a}^{\alpha} \theta_{b}^{\beta} \Lambda_{(A S)}^{a b} \\
& =\Lambda_{(S)}+\Lambda_{(A S)}
\end{aligned}
$$

where

$$
\begin{aligned}
& \Lambda_{(A S)}^{a d}=-\mathcal{J}_{c}^{a} g^{c d}=g^{a e} g^{d c} \theta_{e c}^{-1}=-\Lambda_{(A S)}^{d a} \\
& \Lambda_{(A S)}^{\dagger}=-\Lambda_{(A S)}
\end{aligned}
$$

is anti-symmetric resp. anti-hermitian. It satisfies

$$
P \Lambda=P \Lambda_{(A S)}=\Lambda_{(A S)}, \quad P_{N} \Lambda=P_{N} .
$$

The inverse is given explicitly by

$$
\Lambda^{-1}=P_{N}+\theta_{a} \theta_{b} \theta^{a b} .
$$

We will accordingly define $\Lambda_{\beta}^{\alpha}=\Lambda^{\alpha \beta^{\prime}} \kappa_{\beta^{\prime} \beta}$ etc. Note that the Poisson structure is encoded in $\Lambda$, while the embedding is encoded in $P$. Now consider the Grassmann connection on the projective module $\mathcal{E}_{\gamma}$, given by

$$
\begin{aligned}
\nabla_{\gamma} v & =\Theta^{\alpha} P_{\alpha}^{\beta} d v_{\beta}^{\prime}=\theta \Lambda P d\left(\Lambda^{-1} v\right) \\
\nabla_{\gamma} & =\Lambda \nabla_{g} \Lambda^{-1}
\end{aligned}
$$

Therefore $\nabla_{\gamma}$ is related to $\nabla_{g}$ via the (in general non-orthogonal) transformation $\Lambda$. This is so because $\nabla_{\gamma}$ is compatible with the metric $\gamma$ encoded in $(v, w)_{\gamma}:=v_{\alpha}^{\prime} w_{\alpha}^{\prime}=v_{\alpha}^{\prime} P^{\alpha \beta} w_{\beta}^{\prime}$, while $\nabla_{g}$ is compatible with $g$. The curvature $\nabla_{\gamma}^{2}$ acts on $\mathcal{E}_{\gamma} \cong T^{*} \mathcal{M}$ as follows

$$
\begin{aligned}
\nabla_{\gamma}^{2} v & =\Theta^{\beta} \mathcal{R}_{\beta}^{\alpha}[\gamma] v_{\alpha}^{\prime}=\theta \Lambda \mathcal{R}[\gamma] \Lambda^{-1} v \\
\nabla_{g}^{2} v & =\theta \mathcal{R}[g] v
\end{aligned}
$$


reflecting the fact that the connections $\nabla_{\gamma}$ and $\nabla_{g}$ are related by $\Lambda$. As in the previous section, the coordinate form of the (Grassmann) curvature tensor can be obtained using the frame $\Theta^{\alpha}$

$$
\begin{aligned}
\mathcal{R}_{a b}[\gamma] & =d \Theta_{a} P_{N} d \Theta_{b}^{\dagger}=\Theta_{a} d P_{N} d P_{N} \Theta_{b}^{\dagger} \\
& =\mathcal{R}_{a^{\prime} b^{\prime}}[g] \mathcal{J}_{a}^{a^{\prime}} \mathcal{J}_{b}^{b^{\prime}}=\theta_{a} \Lambda d P_{N} d P_{N} \Lambda^{\dagger} \theta_{b}^{\dagger} .
\end{aligned}
$$

As explained before, the metric (Levi-Civita) connection corresponding to $\gamma$ is given by

$$
\nabla[\gamma]=\nabla_{\gamma}+A[\gamma]
$$

if $A=-A^{\dagger}$ is such that the torsion vanishes,

$$
T[\gamma]=T_{\gamma}+\Theta A[\gamma]=0
$$

To determine $A$, we compute

$$
\begin{aligned}
T_{\gamma} & =d \Theta+m\left(\nabla_{\gamma} \Theta\right)=d \Theta P \\
& =d(\theta \Lambda) P=-\theta d \Lambda P+d \theta P \Lambda \\
& =\Theta\left(d \Lambda^{-1} \Lambda P-\Lambda^{-1} \omega \Lambda\right)
\end{aligned}
$$

using $d \theta P=-\theta \omega(3.14)$ and $P \omega=\omega$. Therefore the torsion $T[\gamma]$ vanishes for

$$
\begin{aligned}
A^{\alpha}{ }_{\beta}[\gamma] & =-P d \Lambda^{-1} \Lambda P+P \Lambda^{-1} \omega \Lambda P+\Theta^{\alpha} B_{\beta}^{(\alpha)} \\
& =P \Lambda^{-1} d \Lambda P+\Lambda^{-1} \omega \Lambda+\Theta^{\alpha} B_{\beta}^{(\alpha)}
\end{aligned}
$$

where $B_{\beta}^{(\alpha)}$ is arbitrary (since $\Theta^{\alpha} \kappa_{\alpha \beta} \Theta^{\beta}=0$ ). This is metric compatible if $A$ is anti-hermitian,

$$
A^{\dagger}=-A
$$

The second term is always anti-hermitian due to (3.48), (3.15) and $\Lambda^{-1} \omega \Lambda=\Lambda_{(A S)}^{-1} \omega \Lambda_{(A S)}$. In particular, the Grassmann connection is torsion-free if $\Lambda$ is unitary, which is evident since then the metrics $g$ and $\gamma$ coincide (3.45).

Conformal rescaling. Now consider the effective metric $G^{a b}=e^{-\sigma} \gamma^{a b}(2.10)$. The above construction can easily be generalized by introducing a suitably rescaled frame

$$
\begin{aligned}
& \tilde{\Theta}_{a}^{\alpha}=e^{-\sigma / 2} \Theta_{a}^{\alpha}=\theta_{b}^{\alpha} \tilde{\mathcal{J}}_{a}^{b}=\theta_{a}^{\beta} \tilde{\Lambda}_{\beta}{ }^{\alpha} \\
& \tilde{\mathcal{J}}^{b}{ }_{a}=e^{-\sigma / 2} \mathcal{J}_{a}^{b}, \quad \tilde{\Lambda}=P_{N}+e^{-\sigma / 2} \Lambda_{(A S)}
\end{aligned}
$$

such that

$$
\tilde{\Theta}_{a}^{\alpha} \tilde{\Theta}_{b}^{\beta} \kappa_{\alpha \beta}=G_{a b}
$$

This leaves the projector $P$ unchanged. However this kind of rescaling is more appropriate after compactification, and we will largely work with $\gamma^{a b}$ in this paper. 


\subsection{Special geometry}

In general, we cannot give an explicit form for the $B_{\beta}^{(\alpha)}$ required for the Levi-Civita connection. We therefore restrict ourselves to a certain class of preferred geometries. More specifically, we consider geometries with

$$
\nabla[g] Q \equiv P d Q P=0
$$

where

$$
\begin{aligned}
Q & :=\Lambda \Lambda^{\dagger}-\mathbb{1}=-\left(\Lambda_{(A S)}^{2}+P\right)=-\theta_{a}\left(\mathcal{J}^{2}+\delta\right)_{b}^{a} g^{b c} \theta_{c}^{\dagger}=\Lambda^{\dagger} \Lambda-\mathbb{1} \\
& =P Q=Q P .
\end{aligned}
$$

$Q$ measures the deviation from $\mathcal{J}$ being an almost-complex structure, in particular $Q=0$ for almost-Kähler geometries (in the Euclidean case). Together with (3.41) and $\nabla Q=\theta \nabla \mathcal{J}^{2} g \theta^{\dagger}$ this implies $\nabla[g] \gamma=\nabla[g]\left(g \mathcal{J}^{2}\right)=0$, so that this condition is equivalent to

$$
\nabla \mathcal{J}^{2}=0, \quad \nabla[g] \equiv \nabla[\gamma] \equiv \nabla[G] \equiv \nabla .
$$

The last equality follows from $\partial \operatorname{det} \mathcal{J}^{2}=0$ together with (2.10). This means that the connections on $\mathcal{M}$ defined by $\gamma$ and $g$ and $G$ are equivalent, which is very reasonable. Now $P d Q P=0$ implies

$$
\begin{aligned}
& 0=P d \Lambda \Lambda^{\dagger} P+P \Lambda d \Lambda^{\dagger} P \\
& 0=P \Lambda^{-1} d \Lambda P+P\left(\Lambda^{-1} d \Lambda\right)^{\dagger} P
\end{aligned}
$$

so that

$$
A_{\Lambda}:=P \Lambda^{-1} d \Lambda P=\Lambda_{(A S)}^{-1} d \Lambda_{(A S)}=-A_{\Lambda}^{\dagger},
$$

using $0=P d P P \equiv \nabla_{g} P$, and the Levi-Civita connection $\nabla[\gamma]$ is obtained for $B_{\beta}^{(\alpha)}=0$. Note that we do not require $\mathcal{J}^{2}=-1$, which is impossible in the Minkowski case due to the inequivalent causal structures of $g$ and $\gamma$. However $\nabla \mathcal{J}^{2}=0$ is compatible with a Minkowski signature, and milden 11 than $\nabla \mathcal{J}=0$. Typically $\mathcal{J}^{2}$ defines an (integrable) decomposition of $T \mathcal{M}$ into rank 2 sub-bundles. Moreover, it is not hard to see that the equations of motion for the Poisson structure $\theta^{a b}$ derived from the bosonic action

$$
S_{\mathrm{YM}} \sim \int d^{2 n} \xi \sqrt{\left|\theta^{-1}\right|} \gamma^{a b} g_{a b}=-\int \Omega^{\wedge n} \operatorname{tr} \mathcal{J}^{-2}
$$

are always satisfied if $\nabla \mathcal{J}^{2}=0$; this will become clear in section 3.7. Moreover, geometries with $\nabla \mathcal{J}^{2}=0$ are not only solutions but are expected to be preferred "ground state" solutions for the Poisson structure. This is true at least for 4-dimensional Euclidean branes, where the bosonic action is positive definite and takes its minimum if and only $\mathcal{J}^{2}=-\delta$ i.e. $Q=0$ [8].

We therefore expect that $\nabla \mathcal{J}^{2}=0$ will always hold at least asymptotically. However in general, $\nabla \mathcal{J}^{2}=0$ might not always be compatible with a given $g$, and matter might lead to short-range perturbations of $\mathcal{J}^{2}$ or $\theta^{a b}$. As observed by Rivelles [17], such perturbations are in fact Ricci-flat at least on $\mathbb{R}^{4}$. Thus we expect that the Poisson structure is adjusted dynamically such that $\nabla \mathcal{J}^{2} \approx 0$, and possible deviations from $\nabla \mathcal{J}^{2}=0$ are suppressed for long distances and could be treated perturbatively. Special geometry should be even less restrictive in the presence of compactified extra dimensions, an compatible with all physically relevant 4-dimensional effective geometries. The dynamics of $\mathcal{J}$ will be studied in section 3.7 .

\footnotetext{
${ }^{11}$ which in turn is milder than e.g. the Kähler condition since $\mathcal{J}^{2} \neq-1$.
} 


\subsection{Curvature and effective gravity}

Let us therefore assume special geometries with $\nabla Q=0$. Then the Levi-Civita connection $\nabla$ is given by

$$
\nabla=\nabla_{\gamma}+A, \quad A=\Lambda_{(A S)}^{-1} d \Lambda_{(A S)}+\omega
$$

using (3.46). After some algebra, we obtain the following expression for the Riemann curvature for $\gamma$ (see appendix (D) using (3.53),

$$
\begin{aligned}
R_{a b}[\gamma] & =\theta_{a}\left(d P_{N} d P_{N}+d \omega+\omega \omega\right) \Lambda \Theta_{b}^{\dagger} \\
& =-R_{a b^{\prime}}[g] \mathcal{J}_{b}^{2 b^{\prime}}=-\left(\mathcal{R}_{a b^{\prime}}[g]+\mathcal{R}_{a b^{\prime}}[\omega]\right) \mathcal{J}_{b}^{2 b^{\prime}}
\end{aligned}
$$

recalling that $\Lambda_{(A S)}^{\dagger}=-\Lambda_{(A S)}$. This also follows from $122 R_{b}^{a}[\gamma] \equiv R_{b}^{a}[g]$, since $\nabla[\gamma]=\nabla[g]$ for special geometries. On the other hand, it follows from (3.67) that $d A_{\Lambda}+A_{\Lambda} A_{\Lambda}=0$, so that the Riemannian curvature for $\gamma$ can be obtained from the Grassmann curvature via

$$
\begin{aligned}
R_{a b}[\gamma] & =\mathcal{R}_{a b}[\gamma]+\Theta_{a} \Lambda^{-1}(d \omega+\omega \omega) \Lambda \Theta_{b}^{\dagger} \\
& =\mathcal{R}_{a^{\prime} b^{\prime}}[g] \mathcal{J}_{a}^{a^{\prime}} \mathcal{J}_{b}^{b^{\prime}}-\theta_{a}(d \omega+\omega \omega) \theta_{b^{\prime}}^{\dagger} \mathcal{J}_{b}^{2 b^{\prime}}
\end{aligned}
$$

using (3.53) in the second line. These are explicit and compact expressions for the effective curvature, which together with the expression (3.32) for $\mathcal{R}_{a b}[g]$ in terms of the currents constitutes a main result of this paper. Comparing the two results (3.69) and (3.68) implies $\mathcal{R}_{a^{\prime} b^{\prime}}[g] \mathcal{J}_{a}^{a^{\prime}} \mathcal{J}_{b}^{-1 b^{\prime}}=-\mathcal{R}_{a b}[g]$, and noting that $\mathcal{R}[\omega]$ satisfies the standard symmetries of the Riemann tensor (e.g. using the explicit form (C.3 $)$ ) we have

$$
\mathcal{R}_{a^{\prime} b^{\prime} ; c d}[g] \mathcal{J}_{a}^{a^{\prime}} \mathcal{J}_{b}^{-1 b^{\prime}}=-\mathcal{R}_{a b ; c d}[g]=\mathcal{R}_{a b ; c^{\prime} d^{\prime}}[g] \mathcal{J}_{c}^{c_{c}^{\prime}} \mathcal{J}_{d}^{-1 d^{\prime}}
$$

Now we compute the Ricci tensor from (3.68):

$$
\operatorname{Ric}_{a c}[\gamma]=\gamma^{b d} R_{a b ; c d}[\gamma]=g^{b d} \mathcal{R}_{a b ; c d}[g]+g^{b d} \mathcal{R}_{a b ; c d}[\omega]=\operatorname{Ric}_{a c}[g]
$$

Consider the two terms separately. For the first term, we use the relation (3.70) as follows

$$
g^{b d} \mathcal{R}_{a b ; c d}[g]=g^{b d} \mathcal{R}_{a^{\prime} b^{\prime} ; c^{\prime} d^{\prime}}[g] \mathcal{J}_{a}^{a^{\prime}} \mathcal{J}_{b}^{-1 b^{\prime}} \mathcal{J}_{c}^{c^{\prime}} \mathcal{J}_{d}^{-1 d^{\prime}}=\gamma^{b d} \mathcal{R}_{a^{\prime} b ; c^{\prime} d}[g] \mathcal{J}_{a}^{a^{\prime}} \mathcal{J}_{c}^{c^{\prime}}
$$

Now we can use the explicit form of $\mathcal{R}[g]$ in terms of the currents is given by (3.32), and together with (3.36) and (3.35) we obtain in NEC

$$
\begin{aligned}
\nabla_{a} J_{b}^{0} & =g_{a b}+K_{a b}^{0}=r \nabla_{a} \partial_{b} r \\
\nabla^{d} J_{d} T_{a c}^{\dagger} & =\gamma^{b d}(T+K)_{b d} T_{a c}^{\dagger}=\gamma^{b d} r \nabla_{b} \partial_{d} r g_{a c}=\left(\gamma^{b d} \nabla_{b} J_{d}^{0}\right) g_{a c} \\
\gamma^{b d} \nabla_{d} J_{b} \nabla_{c} J_{a}^{\dagger} & =\partial_{d}\left(\gamma^{d b} J_{b}\right) \nabla_{c} J_{a}^{\dagger}=\nabla^{d} J_{d} K_{a c}^{\dagger}+\left(\gamma^{b d} \nabla_{b} J_{d}^{0}\right) g_{a c} \\
& =\gamma^{b d} K_{d b} K_{a c}^{\dagger}+\gamma^{b d} K_{d b}^{0} g_{a c}+\left(\gamma^{b d} g_{b d}\right) \nabla_{a} J_{c}^{0} \\
\gamma^{b d} \nabla_{c} J_{b} \nabla_{d} J_{a}^{\dagger} & =\gamma^{b d}\left(T_{b c}+K_{b c}\right)\left(T_{d a}^{\dagger}+K_{d a}^{\dagger}\right) .
\end{aligned}
$$

\footnotetext{
${ }^{12}$ Note that the non-trivial perturbations of $\gamma$ on $\mathbb{R}_{\theta}^{4}$ due to fluctuations of the Poisson structure discussed e.g. in [6, 17] are not compatible with the assumption of special geometry, so there is no contradiction.
} 
using $\left.J^{0}\right|_{p}=0$. To proceed, we assume a compactified brane of the form $\mathcal{M}^{4} \times \mathcal{K} \subset \mathbb{R}^{D}$ where $\mathcal{K}$ has a small radius of scale $r_{K}$, much smaller than any scale $r_{\mathcal{M}}$ associated with the non-compact part. Then the dominant terms are those arising from the extrinsic curvature on $\mathcal{K}$, which is $K_{a b} K_{c d} \sim r_{k}^{-2}$. Therefore we only keep the terms quadratic in $K_{a b}^{\alpha}$ from now on and drop the rest, so that

$$
\begin{aligned}
\gamma^{b d} \nabla_{d} J_{b} \nabla_{c} J_{a}^{\dagger} & =\nabla^{d} J_{d} K_{a c}^{\dagger}+\mathcal{O}\left(\frac{r_{\mathcal{K}}}{r_{\mathcal{M}}}\right) \\
& =-\Lambda_{0}^{-4} T_{c d} \Pi_{e f}^{c d} \theta^{e e^{\prime}} \theta^{f f^{\prime}} K_{e^{\prime} f^{\prime}} K_{a c}^{\dagger}+\mathcal{O}\left(\frac{r_{\mathcal{K}}}{r_{\mathcal{M}}}\right) \\
\gamma^{b d} \nabla_{c} J_{b} \nabla_{d} J_{a}^{\dagger} & =\gamma^{b d} K_{b c} K_{d a}^{\dagger}+\mathcal{O}\left(\frac{r_{\mathcal{K}}}{r_{\mathcal{M}}}\right)
\end{aligned}
$$

using current conservation (4.11). Furthermore, the contributions (C.4) from $g^{a e} \mathcal{R}_{a c ; e f}[\omega]$ are negligible in the same approximation. Then the Ricci tensor for the effective metric is obtained from (3.71) as

$$
\begin{aligned}
\operatorname{Ric}_{a c}[\gamma] & =r^{-2}\left(-\Lambda_{0}^{-4} T_{c d} \Pi_{e f}^{c d} \theta^{e e^{\prime}} \theta^{f f^{\prime}} K_{e^{\prime} f^{\prime}} K_{a^{\prime} c^{\prime}}^{\dagger}-\gamma^{b d} K_{b c^{\prime}} K_{a^{\prime} d}^{\dagger}\right) \mathcal{J}_{a}^{a^{\prime}} \mathcal{J}_{c}^{c^{\prime}} \\
& =r^{-2}\left(\Lambda_{0}^{-4} T_{c d} \Pi_{e f}^{c d} \theta^{e e^{\prime}} \theta^{f f^{\prime}} K_{e^{\prime} f^{\prime}} K_{a^{\prime} c^{\prime}}^{\dagger}+\gamma^{b d} K_{b c^{\prime}} K_{a^{\prime} d}^{\dagger}\right) \mathcal{J}_{c}^{2 c^{\prime}}
\end{aligned}
$$

where the second form follows directly from (3.68). The first line becomes more appealing (and more appropriate for the reduction to 4 dimensions, as explained below) in upper-component notation. Using also $\partial e^{\sigma}=0$, we obtain a compact expression for the Ricci tensor provided $\nabla \mathcal{J}^{2}=0$

$$
e^{2 \sigma} \operatorname{Ric}^{a c}[G]=\operatorname{Ric}^{a c}[\gamma]=-T_{b^{\prime} d^{\prime}} \prod_{b d}^{b^{\prime} d^{\prime}} \mathcal{P}^{b d ; a c}-\Lambda_{0}^{4} g_{b d} \mathcal{P}^{a b ; c d}+\mathcal{O}\left(\frac{r_{\mathcal{K}}}{r_{\mathcal{M}}}\right)
$$

refining 13 the previous results in [20]. However to fully understand the effective gravity on $\mathcal{M}$ we need to understand also the response of the second term $g_{b d} \mathcal{P}^{a b ; c d}$ to matter, which might contain an additional hidden coupling to $T_{a b}$. Therefore this equation does not allow to draw immediate physical conclusions. Nevertheless, the message is that the Ricci tensor is related to the energy-momentum tensor of matter, without invoking an Einstein-Hilbert-type action or quantum effects. The coupling of matter to the Ricci-tensor is mediated by the tensor

$$
\begin{aligned}
\mathcal{P}^{c d ; a b} & =r^{-2} \Lambda_{0}^{-4} \theta^{c c^{\prime}} \theta^{d d^{\prime}} K_{c^{\prime} d^{\prime}} K_{a^{\prime} b^{\prime}}^{\dagger} \theta^{a a^{\prime}} \theta^{b b^{\prime}}=\Lambda_{0}^{-4} \theta^{c c^{\prime}} \theta^{d d^{\prime}} \theta^{a a^{\prime}} \theta^{b b^{\prime}} \partial_{c^{\prime}} \partial_{d^{\prime}} x^{A} \partial_{a^{\prime}} \partial_{b^{\prime}} x_{A} \\
\Pi_{a b}^{c d} & =\delta_{a b}^{c d}-\frac{1}{2(n-1)} \gamma_{a b} \gamma^{c d}
\end{aligned}
$$

which is determined by the extrinsic curvature of the embedding $\mathcal{M}=\mathcal{M}^{4} \times \mathcal{K} \subset \mathbb{R}^{D}$ and the Poisson tensor $\theta^{a b}$. The second form of $\mathcal{P}$ follows from (3.36). Without extrinsic curvature, $\mathcal{P}$ would vanish, and not even Newtonian gravity would arise 14 . The last terms subsume the "mixing terms" which remained mysterious in [20].

The expression (3.76) should be a suitable starting point to study the effective gravity on branes, which will be pursued elsewhere. However we emphasize several points here. First,

\footnotetext{
${ }^{13}$ The sign appears to be inconsistent with [20].

${ }^{14} \mathrm{Of}$ course other mechanisms are conceivable such as induced gravity or holography. However, then the usual fine-tuning problems would arise.
} 
the extrinsic curvature is necessarily large on the compact extra dimensions $\mathcal{K}$, which is transmitted to the non-compact space $\mathcal{M}^{4}$ by the Poisson tensor as in (2.15). In this way, the compactification moduli of $\mathcal{K}$ can play the role of gravitational degrees of freedom for $\mathcal{M}^{4}$, and their origin in the spontaneously broken global symmetries of the matrix model implies that they remain massless 15 . Such Poisson tensors $\theta^{\mu i}$ which relate the compact with the non-compact space naturally arise on compactified brane solutions in matrix models, dubbed split non-commutativity [22]. For example, a spherical compactification $\mathcal{K}=S^{2} \subset \mathbb{R}^{6}$ would lead to $K_{i j} K_{k l}^{\dagger}=r_{\mathcal{K}}^{-2} \delta_{i j} \delta_{k l}$, which is too simple to provide full Einstein gravity. However the $\mathcal{K}$ typically has to rotate along $\mathcal{M}^{4}$ in order to be a solution [22], and there are plenty of more sophisticated compactifications [25]. Moreover, we only need (near-) Einstein gravity in the 4 non-compact direction, and not on $\mathcal{K}$. It remains to be seen if a realistic 4-dimensional gravity can be obtained for suitable compactifications. If so, this could provide a very appealing theory for gravity which is not only well-suited for quantization, but also protected from the usual fine-tuning problems.

It should be clear that this mechanism is completely unavoidable on branes of the structure $\mathcal{M}=\mathcal{M}^{4} \times \mathcal{K} \subset \mathbb{R}^{D}$ in matrix models. It implies a long-range gravity-like force on $\mathcal{M}^{4}$, which would certainly dominate the bulk gravity with its $r^{-8}$ Newton law at long distances. Hence there is no need for 10-dimensional compactification, and the selection of the present type of compactification is a well-defined and predictive question within the matrix model.

Finally, we recall that short-range perturbations with $\nabla \mathcal{J}^{2} \neq 0$ are expected in the presence of matter, as discussed in section 3.7.

Towards 4-dimension gravity. Although the above results apply to any $\mathcal{M} \subset \mathbb{R}^{D}$, we are mainly interested in the low-energy sector on backgrounds of the form $\mathcal{M}=\mathcal{M}^{4} \times \mathcal{K} \subset \mathbb{R}^{D}$. We should therefore perform an appropriate reduction on $\mathcal{K}$, and study the 4-dimensional effective geometry. This reduction is not trivial here, because $\mathcal{K}$ is not perpendicular to $\mathcal{M}^{4}$. As discussed in [20], the effective 4-dimensional metric with upper (!) indices $G^{\mu \nu}$ is obtained from $G^{a b}$ by dropping the extra coordinates 16 , and averaging over $\mathcal{K}$ if necessary

$$
G_{4 D}^{\mu \nu}:=\int_{\mathcal{K}} G^{\mu \nu}
$$

Here we assume that the low-energy physical fields are constant along $\mathcal{K}$ (for the lowest KK modes), which moreover has constant radius as discussed in section 3.8. However, the inverse effective 4-dimensional metric $G_{\mu \nu}^{4 D}$ is in general not such a simple reduction of $G_{a b}$; noncompact coordinates are indicated by Greek letters. We should thus be careful before drawing physical conclusions, but the salient features are expected to survive. In particular, the term

$$
\gamma^{b d} K_{a c} K_{b d}^{\dagger}
$$

is certainly large on $\mathcal{K}$ but should typically vanish on $\mathcal{M}^{4}$, consistent with the fact that the 4dimensional geometry is flat for the basic solutions found in [22, 25], in the absence of matter. In particular, assuming that $\mathcal{P}^{a b ; c d}$ is Lorentz-invariant with respect to the 4-dimensional

\footnotetext{
${ }^{15}$ This is not the case for the radial modes, which were discussed in [20]. These are in fact assumed to be massive here due to the flux on $\mathcal{K}$.

${ }^{16}$ Due to the flux stabilization mechanism, we can assume here that $r_{\mathcal{K}}=$ const as discussed below.
} 
effective metric 17 and assuming that the properly reduced equations (3.76) have the same form, we would indeed obtain the Einstein equations, possibly with additional vacuum contributions due to $g_{n \sigma} \mathcal{P}^{\mu \eta ; \nu \sigma}$. The effective gravitational constant is set by the scales of compactification and $\Lambda_{0}[20]$,

$$
G_{N} \sim r_{\mathcal{K}}^{-2} \Lambda_{0}^{-4}
$$

Although this requires several assumptions about the background (most importantly effective Lorentz invariance of $\mathcal{P}$ ), the message is that an effective gravity similar to Einstein gravity can arise from compactified branes $\mathcal{M}^{4} \times \mathcal{K} \subset \mathbb{R}^{D}$ in matrix models, without an EinsteinHilbert action. The physical meaning of the additional term $g_{\eta \sigma} \mathcal{P}^{\mu \eta ; \nu \sigma}$ remains to be clarified. It might contribute an additional coupling to $T_{\mu \nu}$, and it will probably contribute constant tensors such as $\gamma^{\mu \nu}$ or $(\gamma g \gamma)^{\mu \nu}$. Furthermore, harmonic contributions from $\mathcal{M}^{4}$ may also play a role, cf. [19]. It is tempting to speculate that these modifications of the Einstein equations might manifest themselves as dark matter and/or energy.

\subsection{Energy-momentum conservation}

To understand better possible deviations from $\nabla \mathcal{J}^{2}=0$, we study the tangential degrees of freedom in more detail. These are conveniently captured by the matrix conservation law [6]

$$
0=-i\left[X_{B}, \mathcal{T}^{A B}\right] \sim\left\{x_{B}, \mathcal{T}^{A B}\right\}
$$

where $\mathcal{T}^{A B}$ is the "matrix" energy-momentum tensor. Dropping the contributions of the spinorial (fermionic) matrices $\Psi$, it is given explicitly by

$$
\mathcal{T}^{A B}=\frac{1}{2}\left(\left[X^{A}, X^{C}\right]\left[X^{B}, X_{C}\right]+(A \leftrightarrow B)\right)-\frac{1}{4} \eta^{A B}\left[X^{C}, X^{D}\right]\left[X_{C}, X_{D}\right]
$$

We can split this tensor into geometrical and matter content,

$$
\begin{aligned}
\mathcal{T}^{A B} & =\mathcal{T}_{\text {geom }}^{A B}+\mathcal{T}_{\text {mat }}^{A B}=\partial_{a} x^{A} \partial_{b} x^{B} \theta^{a a^{\prime}} \theta^{b b^{\prime}}\left(T_{a^{\prime} b^{\prime}}^{\text {geom }}+e^{\sigma} \Lambda_{0}^{-4} T_{a^{\prime} b^{\prime}}^{\text {mat }}\right), \\
T_{a b}^{\text {geom }} & =-g_{a b}+\frac{1}{4} \gamma_{a b}\left(\gamma^{c d} g_{c d}\right)
\end{aligned}
$$

and the nonabelian component is essentially the usual energy-momentum tensor, at least for $\theta=$ const. Therefore

$$
\left\{x_{B}, \mathcal{T}_{\text {geom }}^{A B}\right\}=-\left\{x_{B}, \mathcal{T}_{\text {mat }}^{A B}\right\}
$$

describes the back-reaction of matter to the Poisson structure. To understand this, we observe

$$
\begin{aligned}
\left\{x_{B}, \mathcal{T}^{A B}\right\} & =\theta^{c d} \partial_{c} x^{B} \partial_{d}\left(\mathcal{T}^{A D}\right) \eta_{B D} \\
& =\theta^{c d} \partial_{d}\left(\partial_{a} x^{A} \partial_{c} x^{B} \partial_{b} x^{D} \theta^{a a^{\prime}} \theta^{b b^{\prime}} T_{a^{\prime} b^{\prime}}\right) \eta_{B D} \\
& =\theta^{c d} \partial_{d}\left(g_{c b} \theta^{b b^{\prime}} T_{a^{\prime} b^{\prime}} \theta^{a a^{\prime}} \partial_{a} x^{A}\right)
\end{aligned}
$$

\footnotetext{
${ }^{17}$ Lorentz invariance with respect to the full metric on $\mathcal{M}$ is presumably too restrictive, and we do not expect that Einstein gravity is recovered on $\mathcal{M}^{2 n}$. Moreover only a part of the full tensor $\mathcal{P}$ is used after the reduction, so that even the effective sign is not clear at this point.
} 
in any local coordinates. For the geometric contribution, this can be written as

$$
\begin{aligned}
\left\{x_{B}, \mathcal{T}_{\text {geom }}^{A B}\right\} & =\theta^{c d} \partial_{d}\left(\mathcal{J}^{-2}-\frac{1}{4}\left(\operatorname{tr} \mathcal{J}^{-2}\right) \delta\right)_{c}{ }_{c}^{a} \partial_{a} x^{A} \\
& =\theta^{c d}\left(\nabla_{d} \mathcal{J}_{c}^{-2 a}-\frac{1}{4} \partial_{d}\left(\operatorname{tr} \mathcal{J}^{-2}\right) \delta_{c}^{a}\right) \partial_{a} x^{A}
\end{aligned}
$$

(for any torsion-free $\nabla$ ) noting that the transversal contribution vanish, in particular

$$
\gamma^{d b} g_{a^{\prime} b} \theta^{a a^{\prime}} \nabla_{d} \partial_{a} x^{A}=\left(\theta^{d d^{\prime}} g_{d^{\prime} b^{\prime}} \theta^{b b^{\prime}} g_{b a} \theta^{a c}\right) \nabla_{d} \partial_{c} x^{A}=0
$$

since $\theta g \theta g \theta$ is anti-symmetric. Thus (3.86) is purely tangential. For the matter contribution, we can proceed as follows

$$
\begin{aligned}
\Lambda_{0}^{4}\left\{x_{B}, \mathcal{T}_{\text {matter }}^{A B}\right\} & =\frac{e^{\sigma}}{\sqrt{G}} \partial_{d}\left(\sqrt{G} G^{d b} T_{a b} \theta^{a^{\prime} a} \partial_{a^{\prime}} x^{A}\right) \\
& =e^{\sigma}\left(G^{d b}(x) \nabla_{d}[G] T_{b a}-\frac{1}{2} \partial_{a} G^{d b} T_{b d}\right) \theta^{a^{\prime} a} \partial_{a^{\prime}} x^{A}+e^{\sigma} G^{d b} T_{a b} \partial_{d}\left(\theta^{a^{\prime} a} \partial_{a^{\prime}} x^{A}\right) \\
& \stackrel{\text { cons }}{=} e^{\sigma}\left(-\frac{1}{2} T_{b d} \nabla_{a}[g] G^{d b} \mathcal{J}_{e}^{-1 a}+G^{d b} T_{b a} \nabla_{d}[g] \mathcal{J}_{e}^{-1 a}\right) g^{e a^{\prime}} \partial_{a^{\prime}} x^{A}+(\ldots) \nabla[g] \partial x^{A}
\end{aligned}
$$

using the identity [6]

$$
\rho \nabla_{b} \theta^{b c}=\theta^{c b} \partial_{b} \rho, \quad \rho=\sqrt{\mid \theta^{-1 \mid}}
$$

and (E.4) in the appendix. The first two lines hold in any coordinates, and energy-momentum conservation $\nabla^{b}[G] T_{b a}=0$ was assumed in the last line. We choose normal embedding coordinates such that $\partial \partial x^{A}=\nabla[g] \partial x^{A}$ is in the normal bundle, and together with (3.86]) the tangential components give

$$
\theta^{c d} \nabla_{d} \mathcal{J}_{c}^{-2 a}-\frac{1}{4} \theta^{a d} \partial_{d}\left(\operatorname{tr} \mathcal{J}^{-2}\right)=-\frac{e^{\sigma}}{2} \Lambda_{0}^{-4} T_{b d} \nabla_{c}[g] G^{d b} \mathcal{J}_{e}^{-1 c} g^{a e}+e^{\sigma} \Lambda_{0}^{-4} G^{d b} T_{b c} \nabla_{d}[g] \mathcal{J}_{e}^{-1 c} g^{a e} .
$$

Therefore any vacuum geometry with $\nabla \mathcal{J}^{2}=0$ is a solution. Short-range perturbations of $\nabla \mathcal{J}^{2}$ are expected in the presence of matter, which do not significantly contribute to gravity at long distances. To see this, it is better to use the fundamental degrees of freedom given by the Poisson structure and the embedding. Using the identity (E.7), the same conservation law can be written as follows

$$
\gamma^{d a} \nabla_{a}[g] \theta_{b d}^{-1}=\frac{e^{\sigma}}{2} \Lambda_{0}^{-4} T_{b d} \nabla_{c}[g] G^{d b} \theta^{c a} \gamma_{b a}-e^{\sigma} \Lambda_{0}^{-4} G^{d b} T_{b c} \nabla_{d}[g] \theta^{c a} \gamma_{b a}
$$

Since this has the structure of Maxwell equations, the perturbations of $\theta_{b d}^{-1}$ due to matter decay at least as $\frac{1}{r^{2}}$, and therefore do not contribute to gravity at long distances. This is consistent 18 with the equation (3.6) in [20], which was obtained directly from the action.

\footnotetext{
${ }^{18}$ The assumption $\Gamma^{a}=0$ in $[20]$ amounts to $\left\{x_{B}, \mathcal{T}_{\text {geom }}^{A B}\right\}=0$ via $(\underline{\text { E.5 }})$, and therefore follows from $\nabla \mathcal{J}^{2}=0$.
} 


\subsection{Radial equation of motion and flux stabilization}

The equation of motion for the radial mode $r^{2}(x)=x^{A} x_{A}$ can be derived using the identity (3.35), which gives

$$
\frac{1}{2} \square r^{2}=r \square r=\gamma^{a b}\left(g_{a b}+K_{a b}^{0}\right)=\gamma^{a b} g_{a b}-\Lambda_{0}^{-4} T_{c d} \Pi_{a^{\prime} b^{\prime}}^{c d} \theta^{a^{\prime} a} \theta^{b^{\prime} b} K_{a b}^{0}
$$

Since we argued or assumed that $\nabla \mathcal{J}^{2}=0$ to a very good approximation, it follows that $(\gamma g)=-\operatorname{tr} \mathcal{J}^{-2}=$ const. This vanishes if and only if the action is invariant under $x^{A} \rightarrow \alpha x^{A}$, and one may expect that this is preferred upon quantization.

Now consider the case of compactified extra dimensions $M^{4} \times \mathcal{K} \subset \mathbb{R}^{10}$ where $\mathcal{K} \subset \mathbb{R}^{6}$ is compact. We can locally write $\mathbb{R}^{10}=\mathbb{R}^{4} \times \mathbb{R}^{6}$ with $x^{A}=\left(x^{\mu}, y^{i}\right)$ such that the radius is $r_{\mathcal{K}}^{2}=y_{i} y^{i}$, and use the 4 non-compact matrices $x^{\mu}$ as part of the local coordinates $\xi^{a}=\left(x^{\mu}, \xi^{i}\right)$. Then the equation of motion for $r_{\mathcal{K}}$ can be obtained as follows:

$$
\square r^{2}=\left(\gamma^{\mu \nu} \partial_{\mu} \partial_{\nu}\right)\left(x^{\rho} x^{\sigma} \eta_{\rho \sigma}\right)+\square r_{\mathcal{K}}^{2}=2 \gamma^{\mu \nu} \eta_{\mu \nu}+\square r_{\mathcal{K}}^{2}
$$

in NEC. Together with the above we obtain

$$
\begin{aligned}
\frac{1}{2} \square r_{\mathcal{K}}^{2} & =2 \gamma^{i \mu} g_{i \mu}+\gamma^{i j} g_{i j} \\
& =2 \gamma^{i \mu} g_{i \mu}+g_{i j} \theta^{i i^{\prime}} \theta^{j j^{\prime}} g_{i^{\prime} j^{\prime}}=f\left(r_{\mathcal{K}}\right)
\end{aligned}
$$

in vacuum. This is a polynomial in $r_{\mathcal{K}}$ via $g_{i j} \sim r_{\mathcal{K}}^{2}$. If the flux $\theta^{i j}$ on $\mathcal{K}$ does not vanish, then the rhs contains quadratic and quartic terms in $r_{\mathcal{K}}$, and will vanish for a certain radius $r_{0}$ for suitable $\theta^{\mu i}$. The radial perturbations of the compactification $\mathcal{K}$ are then in general stabilized by the flux and (very) massive, so that we can safely set $r_{\mathcal{K}}=$ const at low energies. This is the flux stabilization mechanism in the present context.

\section{Perturbations of the geometry}

Consider a perturbation

$$
x^{A} \rightarrow x^{A}+\delta x^{A}
$$

of some background brane $\mathcal{M}^{2 n} \subset \mathbb{R}^{D}$, defined in terms of matrices $X^{A} \sim x^{A}$ as above. We can certainly describe the most general such deformations in the form

$$
\delta x^{A}=-\sum_{\alpha \neq 0} \epsilon_{\alpha}\left(\lambda^{\alpha} x\right)^{A}+\delta r x^{A}
$$

where $\epsilon_{\alpha}=\epsilon_{\alpha}(x)$ and $\epsilon_{0} \equiv \delta r=\delta r(x)$ are arbitrary functions. This is of course an overparametrization. The corresponding metric perturbation can be written in terms of the currents as

$$
\begin{aligned}
\delta g_{a b} & =-\partial_{a} x \partial_{b}\left(\epsilon_{\alpha}(x) \lambda^{\alpha} x\right)+\partial_{a} x \partial_{b}(\delta r(x) x)+(a \leftrightarrow b) \\
& =J_{a}^{\alpha} \partial_{b} \epsilon_{\alpha}+J_{b}^{\alpha} \partial_{a} \epsilon_{\alpha}+2 \epsilon_{0} g_{a b}
\end{aligned}
$$


since $\lambda^{\alpha \neq 0}$ is anti-symmetric. To clarify the relation with the approach in [20], we can then rewrite this as

$$
\begin{aligned}
\delta g_{a b} & =\nabla_{b}\left(\epsilon^{\alpha} J_{a}^{\alpha}\right)-\epsilon^{\alpha} \nabla_{b} J_{a}^{\alpha}+\epsilon_{0} g_{a b}+(a \leftrightarrow b) \\
& =-2 \epsilon_{\alpha} K_{a b}^{\alpha}+\nabla_{a} V_{b}^{\epsilon}+\nabla_{b} V_{a}^{\epsilon}+2 \epsilon_{0} g_{a b}
\end{aligned}
$$

where $\nabla=\nabla[g]$. Then the vector fields

$$
V_{b}^{\epsilon}=\epsilon^{\alpha} J_{b}^{\alpha}
$$

encode the tangential perturbations, while the extrinsic curvature of $\mathcal{M} \subset \mathbb{R}^{D}$ leads to linearized metric perturbations $-2 \epsilon_{\alpha} K_{a b}^{\alpha}$ due to transversal brane perturbations.

\subsection{Current conservation and matter}

In the presence of matter, the $S O(D)$ rotations also act on the fermions and gauge fields. Rather than trying to derive the generalized currents, we want to incorporate matter as source term for the conservation law of the geometrical current (3.2). We therefore need the variation of the matter action under the local perturbations (4.2) acting only on the geometry defined by the $U(1)$ sector of the matrices $X^{A} \sim x^{A}$, in the presence of fixed matter fields resp. matrices (on-shell). Restricting ourselves to the semi-classical case, matter couples to the background as usual via the effective metric $G$. Therefore the variation of the action under these geometrical $S O(D)$ rotations is simply obtained by the energy-momentum tensor $T_{a b}$ of matter coupled to $\delta G_{a b}$. We choose to work in Darboux coordinates where $\theta^{a b}=$ const is fixed, which is always possible19. Then the variation of the effective metric (2.10) takes the form

$$
\delta G^{a b}=e^{-\sigma} \Pi_{c d}^{a b} \theta^{c c^{\prime}} \theta^{d d^{\prime}} \delta g_{c^{\prime} d^{\prime}}
$$

where

$$
\Pi_{a b}^{c d}=\delta_{a b}^{c d}-\frac{\gamma_{a b} \gamma^{c d}}{2(n-1)}
$$

Then

$$
\begin{aligned}
\delta S_{\mathrm{YM}}+\delta S_{\text {matter }} & =\frac{1}{2(2 \pi)^{n}} \int d^{2 n} x\left(\Lambda_{0}^{4} \sqrt{\theta^{-1}} \gamma^{a b} \delta g_{a b}+\sqrt{G} T_{a b} \delta G^{a b}\right) \\
& =\frac{1}{2(2 \pi)^{n}} \int d^{2 n} x \sqrt{\theta^{-1}}\left(\Lambda_{0}^{4} \gamma^{a b}+T_{c d} \Pi_{a^{\prime} b^{\prime}}^{c d} \theta^{a^{\prime} a} \theta^{b^{\prime} b}\right) \delta g_{a b}
\end{aligned}
$$

where $\delta g_{a b}$ is given by (4.3). Upon partial integration, we obtain the current conservation law in the presence of matter

$$
\begin{aligned}
& \partial_{a}\left(\gamma^{a b} J_{b}^{\alpha}\right)=-\Lambda_{0}^{-4} \partial_{a}\left(T_{c d} \Pi_{a^{\prime} b^{\prime}}^{c d} \theta^{a^{\prime} a} \theta^{b^{\prime} b} J_{b}^{\alpha}\right), \quad \alpha \neq 0 \\
& \partial_{a}\left(\gamma^{a b} J_{b}^{0}\right)=-\Lambda_{0}^{-4} T_{c d} \Pi_{a^{\prime} b^{\prime}}^{c d} \theta^{a^{\prime} a} \theta^{b^{\prime} b} K_{a b}^{0}+\left(\gamma^{a b} g_{a b}\right)
\end{aligned}
$$

\footnotetext{
${ }^{19}$ From the point of view of noncommutative gauge theory on $\mathbb{R}_{\theta}^{2 n}$, this means that all matter fields and $S U(n)$-valued fields are fixed, and only the trace- $U(1)$ scalar fields are perturbed. The latter are interpreted as perturbations of the embedding metric $\delta g_{a b}$.
} 
The second equation follows recalling that $\partial_{a} J_{b}^{0}=g_{a b}+K_{a b}^{0}(\underline{3.30})$, and setting $\left.\left.J^{0}\right|_{p} \sim \partial r\right|_{P}=0$ after a suitable translation. The lhs can be written covariantly using (A.8), and we obtain

$$
\begin{aligned}
e^{\sigma} \nabla^{a}[G] J_{a}^{\alpha} & =\gamma^{a b} K_{a b}^{\alpha}+\mathcal{O}\left(J^{\alpha}\right)=-\Lambda_{0}^{-4} T_{c d} \Pi_{a^{\prime} b^{\prime}}^{c d} \theta^{a^{\prime} a} \theta^{b^{\prime} b} K_{a b}^{\alpha}+\mathcal{O}\left(J^{\alpha}\right), \quad \alpha \neq 0 \\
e^{\sigma} \nabla^{a}[G] J_{a}^{0}-\left(\gamma^{a b} g_{a b}\right) & =\left.\gamma^{a b} K_{a b}^{0}\right|_{p}=-\Lambda_{0}^{-4} T_{c d} \Pi_{a^{\prime} b^{\prime}}^{c d} \theta^{a^{\prime} a} \theta^{b^{\prime} b} K_{a b}^{0}
\end{aligned}
$$

Note that $\mathcal{O}\left(J^{\alpha}\right), \alpha \neq 0$ drops out from the equation (3.76) for the Ricci tensor because it is tangential, while $K_{c d}^{\alpha}$ is transversal. The basic mechanism can now be seen by observing that current conservation (3.3)

$$
\begin{aligned}
e^{\sigma} \nabla^{a}[G] J_{a}^{\alpha} & =\gamma^{a b} K_{a b}^{\alpha}=x \lambda^{\alpha} \square_{G} x, \quad \alpha \neq 0, \\
e^{\sigma} \nabla^{a}[G] J_{a}^{0}-\gamma^{a b} g_{a b} & =\left.\gamma^{a b} K_{a b}^{0}\right|_{p}=x \square_{G} x
\end{aligned}
$$

measures the deviation from harmonicity of the embedding, which couples via $K_{a b}^{\alpha}$ to the energy-momentum tensor, and contributes to $\operatorname{Ric}^{a b}[\gamma]$. This is the same mechanism as in [20].

\section{Conclusion}

In this paper, a formalism for computing the effective curvature of branes in the matrix model is developed. This is done by describing the geometry in terms of an over-complete frame, based on the currents associated with the global $S O(D)$ symmetry of the model. One result is that the effective Ricci tensor has contributions which couple linearly to the energy-momentum tensor. However the coupling is not direct as in general relativity, but somewhat implicit via a coupling tensor $\mathcal{P}$ which depends on the Poisson tensor and the extrinsic curvature of the brane embedding $\mathcal{M} \subset \mathbb{R}^{D}$. An extra term may lead to vacuum solutions which are not Ricci flat. This mechanism is particularly significant for compactified branes $\mathcal{M}=\mathcal{M}^{4} \times \mathcal{K} \subset \mathbb{R}^{D}$, where the coupling $\mathcal{P}$ is always non-vanishing. While the detailed physical consequences depend on the compactification and remain to be clarified, the mechanism clearly leads to a gravity-like long-range force on compactified brane solutions in matrix models, which is not based on the Einstein-Hilbert action. The relation with global symmetries and with non-commutative gauge theory make this mechanism for gravity very attractive for quantization, notably for the maximally supersymmetric IKKT model.

Having confirmed the basic mechanism observed in [20], the tools provided here should allow a more detailed study of the resulting gravity theory. In particular, the additional terms in the geometric equation $(\underline{3.76})$ due to $\mathcal{P}$ need to be understood, the response of $\mathcal{P}$ to matter must be clarified, and suitable compactifications must be found. If the resulting gravity turns out to be viable, this would have far-reaching implications. Since target space does not need to be compactified, the vast landscape of 10-dimensional compactifications and its inherent lack of predictivity could be discarded. It suffices instead to consider lower-dimensional brane compactifications of type $\mathcal{M}^{4} \times \mathcal{K} \subset \mathbb{R}^{10}$, which may also provide the additional structure required for particle physics [26]. Note that there is no contradiction with string theory: the 10-dimensional bulk gravity does indeed arise in a holographic sense. However, bulk gravity is not the dominant mechanism on branes of type $\mathcal{M}^{4} \times \mathcal{K} \subset \mathbb{R}^{10}$ with $B$-field, since the present mechanism leads to a 4-dimensional effective gravity, which is clearly dominant for long distances. Note also that in the matrix model there are a priori no propagating degrees of freedom in the bulk, so that we expect no problem with energy leaking off the brane. This is certainly sufficient motivation for more detailed studies. 
Acknowledgments. Part of this work evolved during an extended visit at the high-energy physics group at CUNY. The hospitality and useful discussions in particular with A. Polychronakos, D. Kabat and D. Karabali and P. Nair are gratefully acknowledged. I also thank J. Zahn and P. Schreivogel for discussions. This work was supported in part by the Austrian Science Fund (FWF) under the contract P24713, and in part by a CCNY/Lehman CUNY collaborative grant.

\section{Appendix A: Conserved currents}

We want to derive the conservation law corresponding to the $S O(D)$ symmetry, which acts as

$$
\delta X^{A}=\lambda_{B}^{A} X^{B}
$$

for some $\lambda \in \mathfrak{s o}(D)$. Consider the corresponding "local" transformation

$$
\delta_{\epsilon} X^{A}=\frac{1}{2} \lambda_{B}^{A}\left\{\epsilon(X), X^{B}\right\} .
$$

The corresponding variation of the action is

$$
\begin{aligned}
\delta S & =\operatorname{Tr} \delta X_{A} \square X^{A}=\frac{1}{2} \operatorname{Tr} \lambda_{A B}\left\{\epsilon(X), X^{B}\right\} \square X^{A} \\
& =\frac{1}{2} \operatorname{Tr} \epsilon(X) \lambda_{A B}\left\{X^{B},\left[X_{C},\left[X^{C}, X^{A}\right]\right]\right\} .
\end{aligned}
$$

Using the identity

$$
\{A,[B, C]\}=[B,\{A, C\}]-\{C,[B, A]\}
$$

this becomes

$$
\begin{aligned}
\delta S & =\frac{1}{2} \operatorname{Tr} \epsilon(X) \lambda_{A B}\left(\left[X_{C},\left\{X^{B},\left[X^{C}, X^{A}\right]\right\}\right]-\left\{\left[X^{C}, X^{A}\right],\left[X_{C}, X^{B}\right]\right\}\right) \\
& =\frac{1}{2} \operatorname{Tr} \epsilon(X) \lambda_{A B}\left(\left[X_{C},\left\{X^{B},\left[X^{C}, X^{A}\right]\right\}\right]\right)
\end{aligned}
$$

as the second term vanishes identically; this reflects the invariance under rigid transformations. This vanishes on-shell for any $\epsilon(X)$, and we obtain the conservation law

$$
\begin{aligned}
{\left[X_{A}, \tilde{J}^{A}\right]=0, \quad \tilde{J}^{C} } & =\frac{1}{2}\left\{\lambda_{A B} X^{A},\left[X^{C}, X^{B}\right]\right\} \sim i \theta^{a b} \partial_{a} X^{C} J_{b}, \\
J_{b} & =\lambda_{A B} x^{A} \partial_{b} x^{B} .
\end{aligned}
$$

This can also be verified directly using the equations of motion (2.5). Note that $\tilde{J}^{A}$ is a tangential vector field on $\mathcal{M} \subset \mathbb{R}^{10}$. In the semi-classical limit, the conservation law amounts to

$$
\begin{aligned}
0 & =\theta^{b c} \partial_{b} X_{A} \partial_{c}\left(\theta^{a e} \partial_{a} X^{A} J_{e}\right)=\theta^{b c} \partial_{c}\left(g_{a b} \theta^{a e} J_{e}\right) \\
& =\gamma^{c e} \partial_{c} J_{e}+\rho^{-1} \partial_{c}\left(\rho \gamma^{c e}\right) J_{e}=e^{\sigma}\left(G^{c e} \partial_{c} J_{e}-\Gamma^{e}[G]\right) J_{e} \\
& =e^{\sigma} \nabla^{e}[G] J_{e}
\end{aligned}
$$


using the identity (․88) for $\rho=\sqrt{\mid \theta^{-1 \mid}}$, and recalling that

$$
-\Gamma^{a}[G]=\frac{1}{\sqrt{|G|}} \partial_{b}\left(\sqrt{|G|} G^{a b}\right)=\rho^{-1} e^{-\sigma} \partial_{b}\left(\rho \gamma^{a b}\right) .
$$

This is the usual covariant conservation law, once again confirming $G$ as the relevant metric. In Darboux coordinates, this conservation law reduces to

$$
e^{\sigma} \nabla^{c}[G] J_{c} \equiv \partial_{c}\left(\gamma^{c e} J_{e}\right)=0 \text {. }
$$

\section{Appendix B: Currents and structure constants}

We observe the following identity for $\mathfrak{s o}(D)$ (resp. $\mathfrak{s o}(1, D-1)$ ) generators

$$
f_{\beta \gamma}^{\alpha} \lambda_{A B}^{\beta} \lambda_{C D}^{\gamma}=2 \eta_{B C} \lambda_{A D}^{\alpha}+2 \eta_{A D} \lambda_{B C}^{\alpha}-2 \eta_{B D} \lambda_{A C}^{\alpha}-2 \eta_{A C} \lambda_{B D}^{\alpha}
$$

where $f_{\beta \gamma}^{\alpha}$ are the structure constants of $\mathfrak{s o}(D)$. This can be established using the basis $\lambda^{\alpha}=\left(\begin{array}{cc}0 & 1 \\ -1 & 0\end{array}\right)$. For the currents $J^{\alpha}$, this implies

$$
\begin{aligned}
f_{\beta \gamma}^{\alpha} J_{b}^{\beta} J_{c}^{\gamma} & =f_{\beta \gamma}^{\alpha}\left(x^{A} \lambda_{A B}^{\beta} \partial_{b} x^{B}\right)\left(x^{C} \lambda_{C D}^{\gamma} \partial_{c} x^{D}\right) \\
& =\partial_{b} r^{2} x^{A} \lambda_{A D}^{\alpha} \partial_{c} x^{D}+\partial_{c} r^{2} x^{C} \lambda_{B C}^{\alpha} \partial_{b} x^{B}-2 r^{2} \partial_{b} x^{B} \lambda_{B D}^{\alpha} \partial_{c} x^{D} \\
& =\partial_{b} r^{2} J_{c}^{\alpha}-\partial_{c} r^{2} J_{b}^{\alpha}-2 r^{2} T_{b c}^{\alpha} \quad(\alpha \neq 0)
\end{aligned}
$$

and therefore

$$
\begin{aligned}
r^{-2} f_{\alpha \beta \gamma} J_{a}^{\alpha} J_{b}^{\beta} J_{c}^{\gamma} & =\partial_{b} r^{2} g_{a c}-\partial_{c} r^{2} g_{a b}-2 \sum_{\alpha \neq 0} J_{a}^{\alpha} T_{b c}^{\beta} \kappa_{\alpha \beta} \\
& =2 r\left(\partial_{b} r g_{a c}-\partial_{c} r g_{a b}-\partial_{b} r g_{a c}+\partial_{c} r g_{a b}-\partial_{a} r g_{b c}\right)+2 r \partial_{a} r g_{b c} \\
& =0 \\
f_{\beta \gamma}^{\alpha} J^{\beta} J^{\gamma} & =2 d r^{2} J^{\alpha}-2 r^{2} d J^{\alpha} .
\end{aligned}
$$

The first identity can also be seen in NEC, and the last identity also holds for $\alpha=0$, where both sides vanish. Combining these, (B.3) implies

$$
d \theta^{\alpha} P^{\alpha^{\prime} \beta} \kappa_{\alpha \alpha^{\prime}}=\frac{d r}{r} \theta^{\beta}=\frac{1}{r} \theta^{0} \theta^{\beta}
$$

which using $\theta^{\alpha} \theta^{\beta} \kappa_{\alpha \beta}=0$ gives (3.14).

\section{Appendix C: Radial curvature}

We compute the curvature contribution due to $\omega$. After a suitable translation (or working in NEC) all first-order derivatives $\partial r$ such as $\omega$ can be dropped, but we must keep the second derivatives:

$$
\begin{aligned}
d\left(r \omega^{\alpha \beta}\right) & =d \theta^{\alpha} P^{(r) \beta}-\theta^{\alpha} d P^{(r) \beta}-d P^{(r) \alpha} \theta^{\beta}-P^{(r) \alpha} d \theta^{\beta} \\
r(P d \omega P)^{\alpha \beta} & =(P d \theta)^{\alpha} P^{(r) \beta}-\theta^{\alpha}(P d P)^{\beta(r)}-(P d P)^{\alpha(r)} \theta^{\beta}-P^{(r) \alpha}(P d \theta)^{\beta} \\
& =-\theta^{\alpha}(P d P)^{\beta(r)}-(P d P)^{\alpha(r)} \theta^{\beta}
\end{aligned}
$$


since $P d \theta=-\theta \omega$ can be dropped. Using $P^{(r) \alpha}=\partial_{a} r g^{a b} \theta_{b}^{\alpha}$ we get

$$
(P d P)^{\beta(r)}=\partial_{f} \partial_{d} r g^{d b} \theta_{b}^{\beta} d x^{f} .
$$

Therefore

$$
\begin{aligned}
R[\omega]_{a c} & =\theta_{a}(d \omega+\omega \omega) \theta_{c}=\theta_{a} d \omega \theta_{c} \\
& =-\theta_{a} \theta_{e}^{\alpha} r^{-1} \nabla_{f} \partial_{d} r g^{d b} \theta_{b}^{\beta} \theta_{c} d x^{e} d x^{f}-\theta_{a} r^{-1} \nabla_{f} \partial_{d} r g^{d b} \theta_{b}^{\alpha} \theta_{c} \theta_{e}^{\beta} d x^{f} d x^{e} \\
& =r^{-2}\left(-g_{a e} \nabla_{f} J_{c}^{0}+g_{c e} \nabla_{f} J_{a}^{0}\right) d x^{e} d x^{f} \\
& =R[\omega]_{a c e f} d x^{e} d x^{f}
\end{aligned}
$$

where $\nabla=\nabla[g]$, using (3.35) and recalling that $\nabla_{a} J_{b}^{0}=\frac{1}{2} \nabla_{a} \partial_{b} r^{2}$. Furthermore, we need the contraction

$$
r^{2} g^{a e} \mathcal{R}_{a c ; e f}[\omega]=2(1-n) \nabla_{f} J_{c}^{0}-g_{c f}\left(g^{a e} \nabla_{e} J_{a}^{0}\right)
$$

\section{Appendix D: Curvature tensor for special geometries}

We note the following identities

$$
\begin{aligned}
P d \Lambda^{-1} P_{N} & =P\left(\mathbb{1}-\Lambda^{-1}\right) d P_{N} \\
P_{N} d \Lambda P & =d P_{N}(\mathbb{1}-\Lambda) P
\end{aligned}
$$

which follow from $\Lambda P_{N}=P_{N}$. Using this and assuming the condition $\nabla Q=0$ such that $B_{\beta}^{(\alpha)}=0$, we obtain using (3.57)

$$
\begin{aligned}
\Theta_{a} A A \Theta_{b}^{\dagger} & =\Theta_{a} \Lambda^{-1} d \Lambda P \Lambda^{-1} d \Lambda \Theta_{b}^{\dagger}=-\Theta_{a} d \Lambda^{-1} P d \Lambda \Theta_{b}^{\dagger} \\
& =-\Theta_{a} d \Lambda^{-1} d \Lambda \Theta_{b}^{\dagger}+\Theta_{a} d \Lambda^{-1} P_{N} d \Lambda \Theta_{b}^{\dagger} \\
& =-\Theta_{a} d \Lambda^{-1} d \Lambda \Theta_{b}^{\dagger}+\Theta_{a}\left(\mathbb{1}-\Lambda^{-1}\right) d P_{N} d P_{N}(\mathbb{1}-\Lambda) \Theta_{b}^{\dagger}
\end{aligned}
$$

To evaluate

$$
\Theta_{a} d A \Theta_{b}^{\dagger}=\Theta_{a}\left(d P \Lambda^{-1} d \Lambda P+P d \Lambda^{-1} d \Lambda P-P \Lambda^{-1} d \Lambda d P\right) \Theta_{b}^{\dagger}
$$

we observe that $P d P_{N} P=0$, which implies the following useful identity

$$
\begin{gathered}
\theta d P=-\theta d P_{N}=-\theta d P_{N} P_{N} \\
d P \theta^{\dagger}=-d P_{N} \theta^{\dagger}=-P_{N} d P_{N} \theta^{\dagger} .
\end{gathered}
$$

This gives

$$
-\Theta_{a} P \Lambda^{-1} d \Lambda d P \Theta_{b}^{\dagger}=\theta_{a} d \Lambda P_{N} d P_{N} \Theta_{b}^{\dagger}=\theta_{a}(\mathbb{1}-\Lambda) d P_{N} d P_{N} \Theta_{b}^{\dagger}
$$

as well as

$$
\Theta_{a} d P \Lambda^{-1} d \Lambda P \Theta_{b}^{\dagger}=-\Theta_{a} d P_{N} P_{N} d \Lambda \Theta_{b}^{\dagger}=-\Theta_{a} d P_{N} d P_{N}(\mathbb{1}-\Lambda) \Theta_{b}^{\dagger}
$$


Then the metric curvature tensor is obtained using (3.53)

$$
\begin{aligned}
R_{a b}[\gamma]= & \theta_{a} \Lambda d P_{N} d P_{N} \Lambda^{\dagger} \theta_{b}^{\dagger}+\Theta_{a}(d A+A A) \Theta_{b}^{\dagger} \\
= & \Theta_{a} d P_{N} d P_{N} \Theta_{b}^{\dagger}+\Theta_{a}\left(\mathbb{1}-\Lambda^{-1}\right) d P_{N} d P_{N}(\mathbb{1}-\Lambda) \Theta_{b}^{\dagger} \\
& +\theta_{a}(\mathbb{1}-\Lambda) d P_{N} d P_{N} \Theta_{b}^{\dagger}-\Theta_{a} d P_{N} d P_{N}(\mathbb{1}-\Lambda) \Theta_{b}^{\dagger}+\Theta_{a} \Lambda^{-1} d \omega \Lambda \Theta_{b}^{\dagger} \\
= & \theta_{a}\left(d P_{N} d P_{N}+d \omega\right) \Lambda \Theta_{b}^{\dagger} \\
= & R_{a b}[g]+\theta_{a} d P_{N} d P_{N} Q \theta_{b}^{\dagger}
\end{aligned}
$$

recalling that $\Lambda \Lambda^{\dagger}=Q+\mathbb{1}$, and dropping $\omega \sim \partial r$ after a suitable translation (or in NEC).

\section{Appendix E: Covariance of conservation laws}

Consider

$$
\begin{aligned}
G^{c a}(x) \nabla_{c}[G] T_{a b} & =G^{c a}(x)\left(\partial_{c} T_{a b}-\Gamma_{c a}^{d} T_{d b}-\Gamma_{c b}^{d} T_{a d}\right) \\
& =G^{c a} \partial_{c} T_{a b}-\Gamma^{d} T_{d b}-G^{c a} \Gamma_{c b}^{d} T_{a d}
\end{aligned}
$$

where

$$
\Gamma^{c}=G^{a b} \Gamma_{a b}^{c}=-\frac{1}{\sqrt{G}} \partial_{d}\left(G^{c d} \sqrt{G}\right)
$$

we can write

$$
G^{c a} \Gamma_{c b}^{d} T_{a d}=\frac{1}{2} G^{c a} G^{\rho d} T_{a d}\left(\partial_{c} G_{e b}+\partial_{b} G_{e c}-\partial_{e} G_{c b}\right)=\frac{1}{2} T^{c e} \partial_{b} G_{e c}
$$

where $T^{c e}=G^{c a} G^{e d} T_{a d}$. Therefore

$$
\begin{aligned}
G^{c a}(x) \nabla_{c}[G] T_{a b} & =G^{c a} \partial_{c} T_{a b}-\frac{1}{2} T^{c \rho} \partial_{b} G_{\rho c}-\Gamma^{\rho} T_{\rho b} \\
& =\frac{1}{\sqrt{G}} \partial_{c}\left(G^{c a} \sqrt{G} T_{a b}\right)+\frac{1}{2} \partial_{b} G^{c a} T_{a c}
\end{aligned}
$$

where the rhs is valid for any connection.

Finally, we recall the identity (see (2.51), (2.53) in [24])

$$
\left\{X_{B}, \mathcal{T}_{\text {geom }}^{A B}\right\}=e^{\sigma} \square_{G} x^{B} \partial_{a} x_{B} \theta^{a e} \partial_{e} x^{A}
$$

and note that

$$
\begin{aligned}
e^{\sigma} \square_{G} x^{b} & =\left\{x^{A},\left\{x_{A}, x^{b}\right\}\right\}=\theta^{a c} \partial_{a}\left(\theta^{b d} g_{d c}\right) \\
& =\theta^{a c} g_{d c} \nabla_{a}[g] \theta^{b d}=-\gamma^{d a} \nabla_{a}[g] \theta_{c d}^{-1} \theta^{b c}
\end{aligned}
$$

Combining these relations gives

$$
\left\{X_{B}, \mathcal{T}_{\text {geom }}^{A B}\right\}=-\gamma^{d a} \nabla_{a}[g] \theta_{b d}^{-1} \gamma^{b e} \partial_{e} x^{A} .
$$




\section{References}

[1] N. Ishibashi, H. Kawai, Y. Kitazawa, A. Tsuchiya, "A Large N reduced model as superstring," Nucl. Phys. B498 (1997) 467-491. [hep-th/9612115].

[2] T. Banks, W. Fischler, S. H. Shenker and L. Susskind, "M theory as a matrix model: A Conjecture," Phys. Rev. D 55 (1997) 5112 [hep-th/9610043].

[3] B. de Wit, J. Hoppe and H. Nicolai, "On the quantum mechanics of supermembranes," Nucl. Phys. B 305 (1988) 545.

[4] S. -W. Kim, J. Nishimura and A. Tsuchiya, "Expanding (3+1)-dimensional universe from a Lorentzian matrix model for superstring theory in (9+1)-dimensions," Phys. Rev. Lett. 108 (2012) 011601 [arXiv:1108.1540 [hep-th]].

[5] H. Steinacker, "Emergent Gravity from Noncommutative Gauge Theory," JHEP 0712 (2007) 049 [arXiv:0708.2426 [hep-th]].

[6] H. Steinacker, "Emergent Gravity and Noncommutative Branes from Yang-Mills Matrix Models," Nucl. Phys. B810 (2009) 1-39. [arXiv:0806.2032 [hep-th]] .

[7] H. Steinacker, "Covariant Field Equations, Gauge Fields and Conservation Laws from Yang-Mills Matrix Models," JHEP 0902 (2009) 044 [arXiv:0812.3761 [hep-th]].

[8] H. Steinacker, "Emergent Geometry and Gravity from Matrix Models: an Introduction," Class. Quant. Grav. 27 (2010) 133001. [arXiv:1003.4134 [hep-th]]

[9] H. Steinacker, "Non-commutative geometry and matrix models," arXiv:1109.5521 [hepth].

[10] N. Seiberg, E. Witten, "String theory and noncommutative geometry," JHEP 9909 (1999) 032. [hep-th/9908142].

[11] D. N. Kabat and W. Taylor, "Linearized supergravity from matrix theory," Phys. Lett. B 426 (1998) 297 [hep-th/9712185]; W. Taylor, "Lectures on D-branes, gauge theory and M(atrices)," In *Trieste 1997, High energy physics and cosmology* 192-271 [hepth/9801182].

[12] J. M. Maldacena, "The Large N limit of superconformal field theories and supergravity," Adv. Theor. Math. Phys. 2 (1998) 231 [hep-th/9711200].

[13] I. Chepelev and A. A. Tseytlin, "Interactions of type IIB D-branes from D instanton matrix model," Nucl. Phys. B 511, 629 (1998) [hep-th/9705120].

[14] D. N. Blaschke and H. Steinacker, "On the 1-loop effective action for the IKKT model and non-commutative branes," JHEP 1110 (2011) 120 [arXiv:1109.3097 [hep-th]].

[15] F. Ferrari, "Emergent Space and the Example of $A d S_{5} \times S^{5}$," arXiv:1207.0886 [hep-th].

[16] L. Susskind, "The Anthropic landscape of string theory," In *Carr, Bernard (ed.): Universe or multiverse?* 247-266 [hep-th/0302219]. 
[17] V. O. Rivelles, "Noncommutative field theories and gravity," Phys. Lett. B558 (2003) 191-196. [hep-th/0212262];

[18] H. S. Yang, "Instantons and emergent geometry," arXiv:hep-th/0608013; H. S. Yang, "On The Correspondence Between Noncommuative Field Theory And Gravity," Mod. Phys. Lett. A 22 (2007) 1119 [arXiv:hep-th/0612231].

[19] H. Steinacker, "On the Newtonian limit of emergent NC gravity and long-distance corrections," JHEP 0912 (2009) 024 [arXiv:0909.4621 [hep-th]].

[20] H. Steinacker, "Gravity and compactified branes in matrix models," JHEP 1207 (2012) 156 [arXiv:1202.6306 [hep-th]].

[21] M. Hanada, H. Kawai and Y. Kimura, "Describing curved spaces by matrices," Prog. Theor. Phys. 114 (2006) 1295 [hep-th/0508211].

[22] H. Steinacker, "Split noncommutativity and compactified brane solutions in matrix models," Prog. Theor. Phys. 126 (2012) 613 [arXiv:1106.6153 [hep-th]].

[23] G. Landi, "An Introduction to noncommutative spaces and their geometry," hepth/9701078.

[24] D. N. Blaschke and H. Steinacker, "Curvature and Gravity Actions for Matrix Models II: The Case of general Poisson structure," Class. Quant. Grav. 27 (2010) 235019 [arXiv:1007.2729 [hep-th]].

[25] A. Polychronakos, H. Steinacker, J. Zahn, in preparation.

[26] A. Chatzistavrakidis, H. Steinacker and G. Zoupanos, "Intersecting branes and a standard model realization in matrix models," JHEP 1109 (2011) 115 [arXiv:1107.0265 [hep-th]]; H. Grosse, F. Lizzi and H. Steinacker, "Gauge Symmetry Breaking in Matrix Models," arXiv:1002.1862 [hep-th]; J. Nishimura and A. Tsuchiya, "Standard Model particles from nonperturbative string theory via spontaneous breaking of Poincare symmetry and supersymmetry," arXiv:1208.4910 [hep-th]. 